University of Zurich

Department of Economics

Working Paper Series

ISSN 1664-7041 (print)

ISSN 1664-705X (online)

Working Paper No. 108

\title{
All-pay Auctions: Implementation and Optimality
}

Stefan Jönsson and Armin Schmutzler

January 2013 


\title{
All-pay Auctions: Implementation and Optimality
}

\author{
Stefan Jönsson and Armin Schmutzler ${ }^{1}$
}

January 2013

\begin{abstract}
This paper analyzes how all-pay auctions with endogenous prizes can be used to provide effort incentives. We show that wide classes of effort distributions can be implemented as equilibrium outcomes of such games. We also ask how all-pay auctions have to be structured so as to induce high expected highest efforts without generating excessive wasteful efforts of losers. All-pay auctions with endogenous prizes can do better than all-pay auctions with fixed prizes in this respect, in particular, when the prize function is approximately linear. We use the results to compare patents and prizes as innovation incentives, and to explore promotion incentives in organizations.
\end{abstract}

Keywords: contests, all-pay auctions, endogenous prizes, implementation

JEL: D44, D43, D02

\footnotetext{
${ }^{1}$ Stefan Jönsson: Department of Economics, University of Zürich, stefan.joensson@econ.uzh.ch. Armin Schmutzler: Department of Economics, University of Zürich and CEPR; armin.schmutzler@econ.uzh.ch. We are grateful to Dirk Bergemann, Subhasish Chowdhury, Andreas Hefti, Kai Konrad, Dan Kovenock, Wolfgang Leininger, Igor Letina, Joao Montez, Georg Nöldeke, Paul Schweinzer, Aner Sela, Ron Siegel, David Wettstein and to seminar audiences at Ben-Gurion University, Exeter, Göttingen (VSP), Hamburg, Munich (IO meeting, Verein für Socialpolitik), Lucerne (ZWE) and Zurich for helpful discussions. Financial support of the Swiss National Science Foundation is gratefully acknowledged.
} 


\section{Introduction}

Many economic institutions rely on competition to provide effort incentives. Contests where only a small subset of players is rewarded with a prize are a prominent example. The recent literature has argued that there are many natural examples where this prize should be regarded as endogenous, influenced by the efforts of the players. Several authors have carefully analyzed the mixed-strategy equilibria of all-pay auctions with endogenous prizes (AEP) for symmetric and asymmetric contestants. ${ }^{2}$ While this literature provides a very general and complete analysis of the equilibrium for any given all-pay auction with endogenous prizes, this paper complements previous work by addressing issues of implementation and optimality.

As an illustration, consider firms engaging in patent races to obtain new products. When firms exert high research efforts, this will usually not only increase the chances of obtaining the patent, but it will also help to improve the quality of the product and thereby the monopoly profits obtained in the product market. Thus, the prize obtained by winning the patent is an increasing function of efforts. The shape of this function cannot be designed freely by a regulator. Technology determines the relation between effort and quality; demand determines the relation between quality and profits. Contests with similar features are pervasive. As we will detail in Section 6, they include beauty contests, where a client invites suppliers to submit project proposals and then procures the project from the supplier who submitted the best proposal, as well as promotion races where employees compete to climb up the career ladder within an organization. We will see that prize functions may be increasing or decreasing in these cases. ${ }^{3}$

Such motivated, we analyze a model of an all-pay auction with complete information, one prize and identical prize functions for all players. ${ }^{4}$ In the first part of the paper, we go further and assume that players also have the same effort cost functions and thus are perfectly symmetric.

\footnotetext{
${ }^{2}$ See, in particular, Kaplan et al. (2003) and Siegel (2009, 2010, 2012).

${ }^{3}$ For further examples, see, e.g., Chowdhury (2010) and Siegel (2012).

${ }^{4}$ The model is similar to those previously analzed by Siegel $(2009,2010,2012)$ and Kaplan et al. (2003).
} 
The first main question is: If we have little information on the details of a competitive environment (e.g., a patent race), except that it is generated by an AEP, what can we say about the possible effort distributions that can result as mixed-strategy equilibria? In other words, what kind of behavior can be implemented by suitable AEP? Our results show that implementation of very general distributions is possible with symmetric players; in other words, the mere fact that a competitive environment corresponds to an AEP imposes hardly any restrictions on the effort distributions. In spite of their simple and special structure, AEPs are thus very rich in terms of the behavior they can induce.

In some contexts, however, there are natural properties of prize functions that can be exploited to obtain stronger restrictions on the outcomes of AEPs. For instance, in the patent race where the prize function reflects expected monopoly profits and its shape depends on demand parameters, the prize function is usually increasing and often concave in effort. With these additional restrictions, we show that only equilibrium distributions with decreasing density functions can arise.

The second main question addresses a central welfare issue in the context of effort incentives. In many competitive situations, the efforts of losers are wasted from an ex-post point of view, that is, they only serve to induce the winner to exert higher efforts. Again, this is the case in patent races where only the winner can introduce the good. We therefore ask under which circumstances AEP induce high expected highest effort without also generating excessive loser efforts. Specifically, we show that the ratio between expected highest efforts and expected average efforts becomes higher for AEP than for all-pay auctions with fixed prizes. Particularly high ratios can be obtained when there is an approximately linear relation between effort and prize or when there are hurdles, that is, reservation values that are necessary to obtain a prize.

Finally, we study the above-mentioned applications. We show that, given the expected average efforts of firms competing to introduce product innovations, patents lead to higher expected product quality than research prizes that compensate for efforts. For promotion contests, we show that a principal who gives a fixed prize for promotion is not necessarily better of if contestants 
also take into account that high efforts may have direct positive effects on their utility.

In Section 2, we discuss the related literature. Section 3 introduces the framework. Section 4 deals with symmetric players, Section 5 with asymmetric players. In Section 6, we provide several applications. Section 7 concludes.

\section{Related Literature}

The literature on contests has been nicely surveyed by Konrad (2009). We thus focus more specifically on AEPs.

Kaplan et al. (2003) have treated a symmetric two-player innovation game with symmetric information, where firms commit to the timing of an innovation. After a simple transformation of variables, the game is almost identical to the AEP with symmetric firms that we consider. ${ }^{5}$ Depending on the prize function, the authors obtain (i) equilibria with randomization on an interval containing zero efforts, (ii) equilibria with an atom at zero and randomization on an interval starting with a positive effort, (iii) more general equilibria with non-connected support.

The asymmetric case has been treated both by Kaplan et al. (2003) and by Siegel $(2009,2010)$ under mutually exclusive assumptions. ${ }^{6}$ In Kaplan et al., the support of the strong player's effort distribution does not contain zero, whereas the support for the weak player contains an atom at zero. In Siegel (2009, 2010), the support of the effort distribution is a compact interval containing the minimal effort, even for the strong player. Also, expected prizes with two players are positive only for the strong player and zero for the weak player. ${ }^{7}$ In some important dimensions, Siegel's analysis is more general than both our paper and Kaplan et al. (2003). He considers more than two players and multiple prizes and he allows for investments that are

\footnotetext{
${ }^{5}$ Essentially, one can define the effort as the inverse of the time needed for innovation.

${ }^{6}$ Kaplan et al. deal only with the two-player case and assume that the prize becomes zero for zero efforts. Siegel assumes that prizes are positive for minimal efforts (and that net prizes are declining in efforts).

${ }^{7}$ This result has been generalized in Siegel (2012).
} 
conditional on winning or losing. Also, he allows for very general types of prize and cost asymmetries. ${ }^{8}$

Contrary to the existing literature, our main concern is to analyze which CDFs can be implemented as MSE of AEPs and which prize functions induce high expected highest efforts. To this end, we allow for prize functions that are compatible with the assumptions of Siegel as well as Kaplan et al. Accordingly, we obtain both equilibria where small positive efforts are avoided by the strong player (as in Kaplan et al. 2003) and equilibria where they are played (as in Siegel 2009, 2010). ${ }^{9}$ We also provide a recursive formula for MSE with arbitrarily many connected components in the symmetric case. Though this possibility arises in Kaplan et al. (2003) and Siegel (2012), there is no analogous result there.

A more broadly related literature allows for asymmetric information in AEP. Kaplan et al. (2002) derive comparative static results when the prize function differs across player types, which are private information. In a similar setting, Cohen et al. (2008) consider the optimal prize function for a principal who cares about maximizing total effort or expected highest effort (net of expected prizes). Interestingly, the optimal prize function can be decreasing in efforts for suitable prize functions when there are many participants. We do not deal with asymmetric information, because we want to focus on which effort distributions can be generated endogenously rather than as a reflection of type distributions.

Moldovanu and Sela (2001) is a paper that does not deal with AEP, but deserves to be mentioned. It also analyzes the question of maximizing the expected highest effort, but in a setting with fixed prizes and asymmetric information. Instead of asking how the shape of the prize function affects expected highest effort, the authors address the role of the number of prizes.

\footnotetext{
${ }^{8}$ Chowdhury (2010) analyzes a modified all-pay auction where prizes are fixed, but not guaranteed even for a player who has exerted effort. If the probability that a prize is actually distributed is increasing in the effort of the high-effort player, the game is isomorphic to an AEP with endogenous prizes.

${ }^{9}$ Several authors have also considered the case that prizes depend not only on the effort of the winner, but also on the loser (Skaperdas 1992, Chung 1996, Baye et al. 2010); Sacco and Schmutzler (2008) provide an experimental analysis.
} 


\section{$3 \quad$ Set-Up and Terminology}

We consider a game with complete information. Risk-neutral players $i \in \mathcal{I} \equiv$ $\{1,2, \ldots, n\}$ simultaneously choose efforts $x_{i}$ from $X_{i}=\mathbb{R}^{+}$at $\operatorname{costs} K_{i}\left(x_{i}\right)$.

Assumption A1: $K_{i}\left(x_{i}\right)=k_{i} x_{i}$ for positive constants $k_{i}>0$ such that $k_{1} \leq k_{2} \leq \ldots \leq k_{n}=1 .^{10}$

Let $\mathbf{x}_{-i}=\left(x_{1}, \ldots, x_{i-1}, x_{i+1}, \ldots, x_{n}\right)$. Player $i$ wins a prize of size $a\left(x_{i}\right)$ with probability $p\left(x_{i}, \mathbf{x}_{-i}\right)=1$ if $x_{i}>\max _{j \neq i} x_{j}$ and $p\left(x_{i}, \mathbf{x}_{-i}\right)=0$ if $x_{i}<$ $\max _{j \neq i} x_{j}$. If several players exert the same highest effort, each of them wins with the same probability. The expected payoff of player $i$ is

$$
\pi_{i}\left(x_{i}, \mathbf{x}_{-i}\right)=p\left(x_{i}, \mathbf{x}_{-i}\right) a\left(x_{i}\right)-k_{i} x_{i}
$$

Except where otherwise mentioned, we maintain the following assumptions on the prize function $a\left(x_{i}\right), a: \mathbb{R}^{+} \rightarrow \mathbb{R}^{+}$.

Assumption A2: $a\left(x_{i}\right)$ is (i) right continuous on $\mathbb{R}^{+}$, and (ii) it is continuously differentiable to the right on $(0, \infty)$.

Assumption A3: (i) $\forall i \in \mathcal{I} \exists r_{i}>0$ s.t. $a\left(r_{i}\right)=k_{i} r_{i}$ and $a\left(x_{i}\right)<$ $k_{i} x_{i}$ for $x_{i}>r_{i}$.

(ii) $a\left(x_{i}\right)$ is continuously differentiable in an open neighborhood of $r_{i}$, with $a^{\prime}\left(r_{i}\right)<k_{i}$ for $i=1, \ldots, n$.

We apply the notation $a^{\prime}\left(x_{i}\right)$ to denote the right derivative even where no left derivative exists. We call prize functions satisfying A2 and A3 admissible. The left panel in Figure 1 depicts examples. A3(i) corresponds to the finite reach assumption, which is required for existence of an MSE. ${ }^{11}$ A3(ii) rules out degenerate cases where the prize function touches the cost function from below at $r_{i}$ (as in the right panel of Figure 1).

\footnotetext{
${ }^{10}$ The requirement that $\max \left(k_{1}, \ldots, k_{n}\right)=1$ is a normalization that guarantees that efforts can be identified with monetary costs in the symmetric case.

${ }^{11}$ See Siegel $(2009,2010)$; similarly in Kaplan et al. (2003).
} 
Definition 1 An all-pay auction with endogenous prize $(\boldsymbol{A E P})$ is a simultaneous game with players $i \in \mathcal{I} \equiv\{1,2, \ldots, n\}$, action spaces $X_{i}=\mathbb{R}^{+}$ and payoffs functions $\pi_{i}\left(x_{i}, \mathbf{x}_{-i}\right)$ given by (1), where $K_{i}\left(x_{i}\right)$ satisfies $A 1$ and a: $\mathbb{R}^{+} \rightarrow \mathbb{R}^{+}$satisfies A2 and A3.
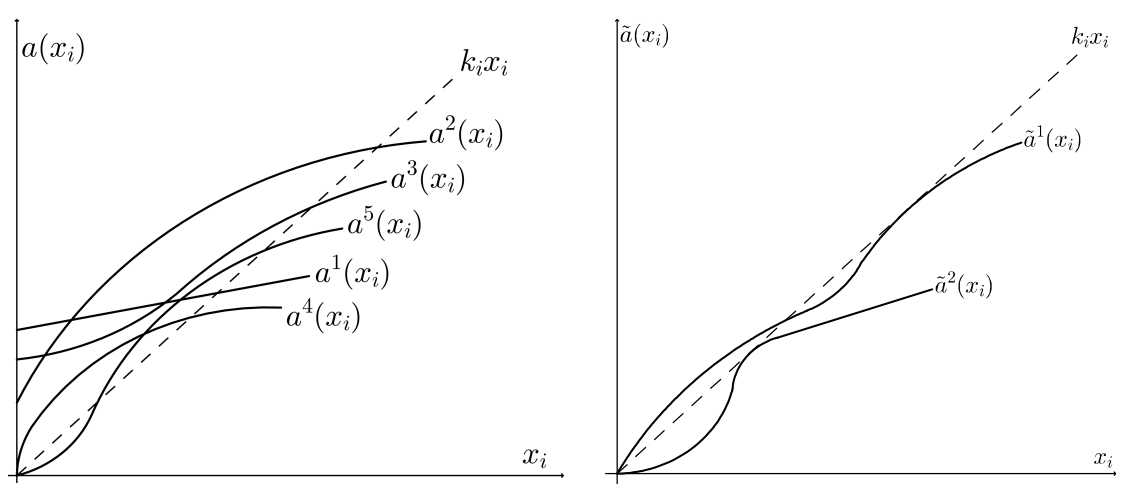

Figure 1: Admissible and non-admissible prize functions

All our statements refer to AEPs. Contrary to the setting with fixed prizes, A1 is not a strong restriction with endogenous prizes. ${ }^{12}$ An AEP with some arbitrary effort measure $x_{i}$ and a corresponding monotone increasing (potentially non-linear) cost function can always be transformed into an equivalent all-pay auction satisfying A1. ${ }^{13}$ In Section 4, we impose the additional restriction of symmetric cost functions.

(SYM): $k_{i}=1$ for all players $\left(K_{i} \equiv K\right)$.

As Siegel (2009, 2010), we use the following concepts for an AEP.

Definition 2 (i) The reach of player $i$ is $r_{i}$ as in A3(i).

\footnotetext{
${ }^{12}$ Moldovanu and Sela (2001) analyze how the optimal number of prizes depends on the second derivatives of the effort cost functions.

${ }^{13}$ Consider the transformation $x_{i}^{T}=K_{i}\left(x_{i}\right)$. Let $K^{-1}\left(x_{-i}\right)$ $\equiv$ $\left(K_{1}^{-1}\left(x_{1}\right), \ldots, K_{i-1}^{-1}\left(x_{i-1}\right), K_{i+1}^{-1}\left(x_{i+1}\right), \ldots, K_{n}^{-1}\left(x_{n}\right)\right)$. Then consider the all-pay auction with endogenous prize defined by $a^{T}\left(x_{i}^{T}\right)=a\left(K^{-1}\left(x_{i}^{T}\right)\right)$ and $K_{i}^{T}\left(x_{i}^{T}\right)=x_{i}^{T}$, where firms choose efforts $x_{i}^{T}$, the winner obtains $a^{T}$ and the cost function is $K_{i}^{T}$. This AEP has the same economic content, except for the renormalization of variables. Nevertheless, the transformation leads to important changes in the interpretation of results. For instance, if the principal cares about the expectation of the effort $x_{i}$ in the all-pay auction with a quadratic cost function, his objective function will be the expected root of the effort $\widetilde{x}_{i}$ after the transformation.
} 
(ii) The threshold $T$ of the game is $r_{2}$.

(iii) The power of player $i$ is $w_{i}=\max \left\{a(T)-k_{i} T, 0\right\}$.

The reach is higher for players with lower effort costs; thus, it is decreasing in $i$. Actions above the threshold are dominated for $i=2, \ldots, n$; unless dominated strategies are played by the opponent, the strongest player can secure himself the prize by choosing efforts just above the threshold. With (SYM), actions above the threshold are dominated for all players.

As pure strategies often do not exist in AEP, we focus on mixed strategies.

Definition 3 (i) A mixed strategy of player $i$ in an AEP is given by a cumulative distribution function $(C D F) F_{i}: \mathbb{R} \rightarrow \mathbb{R}$ such that $F_{i}\left(x_{i}\right)=0$ for $x_{i}<0 .{ }^{14}$

(ii) A mixed strategy profile $\mathbf{F}$ consists of mixed strategies $F_{1}, \ldots, F_{n}$ on $X_{1}, \ldots, X_{n}$.

(iii) A mixed strategy profile $\mathbf{F}$ is a mixed strategy equilibrium (MSE) if each $F_{i}$ maximizes (1) given $\mathbf{F}_{-i}$.

(iv) The support $\mathcal{S}_{i}$ of a mixed strategy $F_{i}$ is defined as the set $\left\{x_{i} \in X_{i} \mid F_{i}\left(x_{i}+\varepsilon\right)-F_{i}\left(x_{i}-\varepsilon\right)>0 \forall \varepsilon>0\right\}$. We write $\mathcal{S}=\mathcal{S}_{i}$ when $\mathcal{S}_{i}$ is independent of $i$.

The following definition is central.

Definition 4 An admissible prize function a(.) implements a profile $\mathbf{F}$ of $\boldsymbol{C D F s}$ if $\mathbf{F}$ is an MSE for the AEP given by $a($.$) . When a(.) implements$ a symmetric $\mathbf{F}=(F, \ldots, F)$, we also say that a $($.$) implements the \boldsymbol{C D F} F$.

Under symmetry, the following types of MSE are of particular interest.

Definition 5 Suppose SYM holds.

(i) A symmetric MSE with CDF $F$ is an interval equilibrium without atoms if it admits a density $f$ which is positive only on $\mathcal{S}=[0, T]$.

(ii) A symmetric MSE with CDF F is called an interval equilibrium with

\footnotetext{
${ }^{14}$ Equivalently, one can define a mixed strategy by a probability measure $P$ on $X_{i}$; the corresponding $F$ is given by $F=P\left[0, x_{i}\right]$. When a mixed strategy is given by $F$, the corresponding $P$ is induced by $P\left(x_{i}^{1}, x_{i}^{2}\right]=F\left(x_{i}^{2}\right)-F\left(x_{i}^{1}\right)$.
} 
atoms if it has support $[0, T]$, but has an atom.

(iii) A symmetric MSE with CDF $F$ is a hurdle equilibrium if $\mathcal{S}=\{0\} \cup$ $\left[H_{S}, T\right]$ for some $H_{S}>0$, the hurdle.

The following definitions will play an important role.

Definition 6 (i) a $\left(x_{i}\right)$ satisfies decreasing (average) effort productivity $(\boldsymbol{D E P})$ if $\frac{a\left(x_{i}\right)}{x_{i}}$ is strictly decreasing on $[0, T]$.

(ii) The effort hurdle $\left(H_{S}^{*} \equiv H_{S}^{*}(a)\right)$ is the minimal $\underline{x_{i}}$ such that $\frac{a\left(x_{i}\right)}{x_{i}}$ is strictly decreasing on $\left(\underline{x_{i}}, T\right]$.

$H_{S}^{*}$ exists by A3(ii). If DEP holds, $H_{S}^{*}=0$. DEP implies that $a\left(x_{i}\right)>0$ for $x_{i} \in(0, T]$, because $\frac{a(T)}{T}=1>0$. If $a\left(x_{i}\right)$ is differentiable, $H_{S}^{*}$ is the minimal $\underline{x}_{i}<T$ such that $a^{\prime}\left(x_{i}\right)<\frac{a\left(x_{i}\right)}{x_{i}}$ for all $x_{i} \in\left(\underline{x}_{i}, T\right]$.

\section{Symmetric Case}

The main result for symmetric players, Proposition 3, shows which CDFs can be implemented as symmetric interval equilibria. We also provide general implementation results for distributions without small positive efforts in the support (Propositions 5 and 6). Moreover, we investigate which prize functions implement MSE with high expected highest efforts. As a preparation, we characterize the MSE for symmetric prize functions under DEP in Proposition 1, and we also discuss uniqueness (Proposition 2). In Section 4.4, we show that additional MSE arise if DEP is relaxed.

\subsection{Existence, Characterization and Uniqueness}

We first focus on interval equilibria; more equilibria will be discussed in Section 4.4. We distinguish between three mutually exclusive cases, C1-C3.

(C1) (i) $a(0)>0$ and (ii) DEP holds.

Siegel (2010) uses C1(i), and instead of C1(ii) he assumes declining net prizes $a\left(x_{i}\right)-x_{i}$, as for $a^{1}\left(x_{i}\right)$ in the left panel of Figure $1 .{ }^{15}$ For differentiable

\footnotetext{
${ }^{15}$ Similar assumptions are used in Siegel (2009).
} 
$a\left(x_{i}\right)$, declining net prizes imply $a^{\prime}\left(x_{i}\right)<1$ and thus $a^{\prime}\left(x_{i}\right)<\frac{a\left(x_{i}\right)}{x_{i}}$ for $a\left(x_{i}\right) \geq$ $x_{i}$, so that DEP holds. ${ }^{16} \mathrm{C} 1$ also holds for strictly concave functions such as $a^{2}\left(x_{i}\right)$ in Figure 1 even if they do not satisfy declining net prizes. Some nonconcave functions such as $a^{3}\left(x_{i}\right)$ also satisfy $\mathrm{C} 1$. The following alternative condition allows for prizes that are zero when there is no effort.

(C2) (i) $a(0)=0$, (ii) $a^{\prime}(0) \equiv \lim _{x_{i \rightarrow 0}} a^{\prime}\left(x_{i}\right)=\infty$ and (iii) DEP holds.

This obviously excludes declining net prizes, but it includes concave prize functions such as $a^{4}\left(x_{i}\right)$ in Figure $1 .^{17}$

Finally, we allow for prize functions with $a(0)=0$ and finite slope at zero.

(C3) (i) $a(0)=0$, (ii) $a^{\prime}(0)<\infty$ and (iii) DEP holds.

We can now characterize the MSE.

Proposition 1 Suppose SYM and DEP hold.

(i) An MSE exists for which

$$
\left[F\left(x_{i}\right)\right]^{n-1}=\frac{x_{i}}{a\left(x_{i}\right)} \text { for } 0<x_{i} \leq T
$$

(ii) If C1 and C2 hold, the equilibrium CDF is atomless; if C3 holds, there is an atom at 0 with mass $\left(\frac{1}{a^{\prime}(0)}\right)^{\frac{1}{n-1}}$.

Intuitively, the indifference condition for an MSE requires that players are exactly compensated for expected efforts. Thus, (2) must hold. The proof shows that the conditions of the proposition suffice to make sure that (2) defines an MSE.

We rule out other symmetric MSE than those in Proposition 1 if DEP holds. Moreover, interval equilibria cannot exist if DEP does not hold.

\footnotetext{
${ }^{16}$ This implication holds because with $a^{\prime}\left(x_{i}\right)<1$ the net prize $a\left(x_{i}\right)-x_{i}$ has at most one fixed point and $a\left(x_{i}\right)>x_{i}$ if and only if $x_{i}$ lies to the left of this point. Thus $a^{\prime}\left(x_{i}\right)<1<\frac{a\left(x_{i}\right)}{x_{i}}$ whenever $a\left(x_{i}\right)>x_{i}$.

${ }^{17}$ Specifically, the class $a(x)=\alpha x^{\gamma}$ with $\alpha>0$ and $\gamma<1$ satisfies C2. Note that $\alpha x^{\gamma}$ is not necessarily monotone increasing (only if $\gamma \in(0,1)$ ).
} 


\section{Proposition 2 Suppose $S Y M$ holds.}

(i) If DEP holds, the MSE described in Proposition 1 is unique.

(ii) If DEP does not hold, the AEP does not have an interval equilibrium.

Thus, an AEP has an interval equilibrium if and only if DEP holds.

\subsection{Implementation Results}

We now consider the converse problem. Suppose given a CDF $F$, does there exist an admissible prize function which implements this CDF? We provide a complete characterization of the CDFs that can be implemented as interval equilibria of a suitable AEP. From Propositions 1 and 2, any candidate prize function with MSE $F$ and support $[0, T]$ must satisfy

$$
\begin{array}{ccc}
a^{F}\left(x_{i}\right)=\frac{x_{i}}{\left[F\left(x_{i}\right)\right]^{n-1}} & \text { for } & 0<x_{i} \leq T \\
a^{F}(0)=\lim _{x_{i} \rightarrow 0} & \text { for } & x_{i}=0
\end{array} .
$$

Also by Propositions 1 and 2, a prize function satisfying (3) is admissible and yields the equilibrium $\mathrm{CDF} F$ if and only if $\mathrm{A} 2, \mathrm{~A} 3$ and, in addition, $\mathrm{C} 1$ or $\mathrm{C} 2$ hold. This leads to the following result.

Proposition 3 Suppose a CDF F has a density $f$ such that $\left\{x_{i} \mid f\left(x_{i}\right)>0\right\}=$ $[0, C]$ for $C>0$.

(i) F can be implemented as interval equilibrium without atoms if and only if the following conditions both hold:

$$
\begin{aligned}
& \lim _{x_{i} \rightarrow 0} F\left(x_{i}\right)^{n-2} f\left(x_{i}\right)>0 . \\
& \lim _{x_{i} \rightarrow 0} F\left(x_{i}\right)^{n-2} f\left(x_{i}\right)<\infty \text { or } \lim _{x_{i} \rightarrow 0} \frac{F\left(x_{i}\right)-x_{i}(n-1) f\left(x_{i}\right)}{F\left(x_{i}\right)^{n}}>0
\end{aligned}
$$

(ii) If (4) holds, but (5) does not, then $F$ can be implemented as interval equilibrium with atom at zero. 
The proof shows that (4) guarantees that $a^{F}$ is admissible. ${ }^{18}$ (5) makes sure that $a^{F}$ satisfies $\mathrm{C} 1$ or $\mathrm{C} 2$. Hence, $\mathbf{F}=(F, \ldots, F)$ really is an interval equilibrium without atoms in case (i) and with atoms in case (ii).

Additional properties of $a\left(x_{i}\right)$ require further restrictions on $F$ :

Corollary 1 Under the conditions of Proposition 3, a $\left(x_{i}\right)$ can be chosen to be strictly increasing if and only if

$$
\frac{f\left(x_{i}\right)}{F\left(x_{i}\right)}<\frac{1}{(n-1) x_{i}} \forall x_{i} \in[0, C]
$$

Proposition 3 is very powerful if $n=2$.

Corollary 2 If $n=2$, any $C D F F$ with a finite and strictly positive density on $[0, C]$ for $C>0$ can be implemented as interval equilibrium without atoms for an admissible prize function $a\left(x_{i}\right)$. If $f$ is differentiable, this prize function is

(i) strictly concave if and only if $\forall x_{i} \in \mathbb{R}^{+}$

$$
\frac{f^{\prime}\left(x_{i}\right)}{f\left(x_{i}\right)}+\frac{2}{x_{i}}-2 \frac{f\left(x_{i}\right)}{F\left(x_{i}\right)}<0
$$

(ii) strictly increasing if and only if $\frac{f\left(x_{i}\right)}{F\left(x_{i}\right)}<\frac{1}{x_{i}} \forall x_{i} \in \mathbb{R}^{+}$;

(iii) not strictly increasing and concave unless $f^{\prime}\left(x_{i}\right)<0 \forall x_{i} \in \mathbb{R}^{+}$.

Condition (ii) requires $\frac{f\left(x_{i}\right)}{F\left(x_{i}\right)}$ to be smaller than for the uniform distribution, which is the MSE for the AEP with fixed prize.

Even when the density is not finite, implementation is often possible for $n=2$. For instance, in Section 4.3 we show that power distributions can be obtained as MSE for suitable prize functions. ${ }^{19}$

For $n>2$, implementation by admissible prize functions is only possible if the density is unbounded.

Corollary 3 Let $n>2$. A CDF $F$ with density $f$ cannot be implemented as an interval equilibrium if $\lim _{x_{i} \rightarrow 0} f\left(x_{i}\right)<\infty$.

\footnotetext{
${ }^{18}$ If (4) does not hold, the candidate function does not converge to a finite value as efforts approach zero, so that A2 is violated.

${ }^{19}$ In this case, the first condition in (5) is violated, but the second one is not.
} 


\subsection{Optimality}

How does the prize function affect expected average and highest efforts? We compare prize equivalent AEP, that is, AEP resulting in the same expected prize payments in the MSE.

Expected Average Efforts In any MSE of a symmetric AEP containing 0 in the support, players must receive expected prizes that exactly compensate their effort costs. Thus, the following result is immediate from A1.

Proposition 4 If SYM holds, any two prize-equivalent AEPs yield the same expected effort.

Expected Highest Efforts As argued in the introduction, it is often desirable in applications to induce a high expected highest effort (first-order statistic), while keeping average efforts low. For $n=2$, this amounts to a high ratio

$$
\rho_{2}=\frac{2 \int_{0}^{C} x_{i} f\left(x_{i}\right) F\left(x_{i}\right) d x_{i}}{\int_{0}^{C} x_{i} f\left(x_{i}\right) d x_{i}} .
$$

Because $F\left(x_{i}\right) \leq 1, \rho_{2} \leq 2$. To understand how close $\rho_{2}$ can be to this maximum, we focus on a rich class of examples. For parameters $\alpha>0$ and $\gamma<1$, we consider the prize functions

$$
a\left(x_{i}\right)=\alpha x_{i}^{\gamma}
$$

For $\gamma=0,(8)$ defines a fixed prize all-pay auction. The limit case $\gamma=1$ is a linear prize function. More generally, $\gamma$ corresponds to higher sensitivity of prizes to efforts: The ratio between the prize of a player that wins with effort $x_{H}$ and the prize of a player that wins with effort $x_{L}<x_{H}$ is increasing in $\gamma$.

As (8) satisfies A2-A3 and C2, it defines an AEP if $K\left(x_{i}\right)=x_{i}$. Thus, by Propositions 1 and 2, the unique symmetric MSE is the power distribution

$$
F\left(x_{i}\right)=P^{\alpha ; \gamma}\left(x_{i}\right) \equiv\left\{\begin{array}{ccc}
\frac{1}{\alpha} x_{i}^{1-\gamma} & \text { for } & 0<x_{i} \leq \alpha^{\frac{1}{1-\gamma}} \\
1 & \text { for } & x_{i} \geq \alpha^{\frac{1}{1-\gamma}}
\end{array}\right.
$$


Standard calculations show that prize equivalence implies $\alpha^{\frac{1}{1-\gamma}} \frac{1-\gamma}{2-\gamma}=C$, where $C$ is the expected cost (effort); examples of such equivalence classes are depicted in Figure A1. Solving for $\alpha$ yields

$$
\alpha=\left(C \frac{2-\gamma}{1-\gamma}\right)^{1-\gamma}
$$

Inserting (10) in (8), $a\left(x_{i} ; \gamma\right)=\left(C \frac{2-\gamma}{1-\gamma}\right)^{1-\gamma} x_{i}^{\gamma}$ for $\gamma<1$ gives a class of prize equivalent AEP. Several members of a class of prize equivalent AEP are depicted in Figure A2 for $C=1$ and $\gamma=-0.1,0,0.5$ and 0.8 .
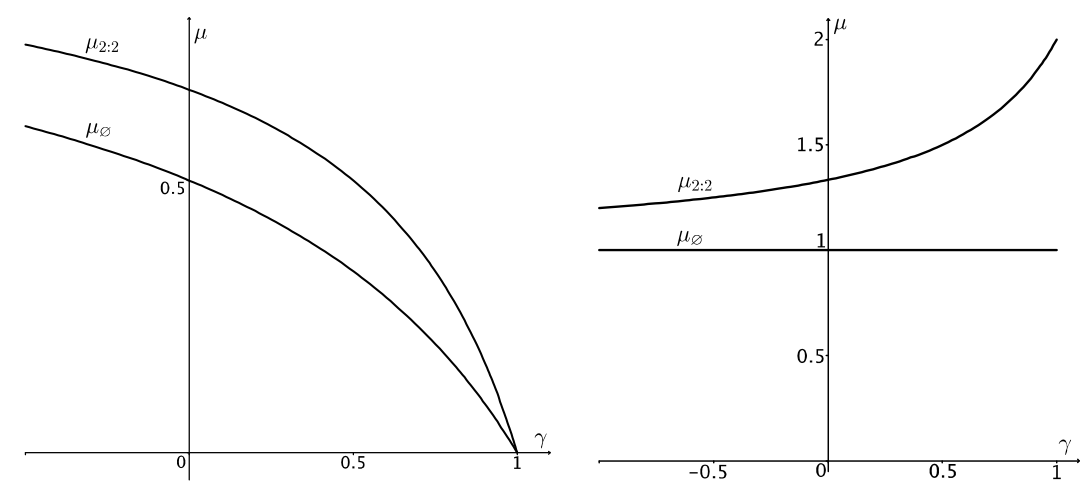

Figure 2: Expected average and highest effort in symmetric game as a function of $\gamma$ for $a\left(x_{i}\right) \equiv 1$ (left panel) and within a class of prize-equivalent APA (right panel)

Using (9), any class of prize equivalent AEP given by (10) corresponds to a class of equilibrium CDFs $\left(C \frac{2-\gamma}{1-\gamma}\right)^{\gamma-1} x_{i}^{1-\gamma}$. The upward-sloping curve in the right panel in Figure 2 shows the equilibrium expected highest effort $\mu_{2: 2}$ as a function of $\gamma$ within a class of prize equivalent AEP: Thus, within the class of incentive systems that lead to power distributions, the resulting expected highest effort approaches the theoretical maximum of twice the expected average effort as $\gamma \rightarrow 1$. For completeness, the panel also contains the expected average effort $\mu_{\varnothing}$, which is independent of $\gamma$ within each class of prize-equivalent AEP (consistent with Proposition 4). The left panel in Figure 2 displays expected highest and average efforts as decreasing functions of $\gamma$ for $\alpha=1$. The difference to the right panel arises because, as $\gamma$ increases, $\alpha$ must increase to guarantee prize equivalence. A ceteris paribus increase 
in $\gamma$ has negative effects on efforts, but the concomitant increase in $\alpha$ has positive effects. Thus, we obtain:

Result: For $n=2$, the maximum possible expected highest effort level within the class $a\left(x_{i}\right)=\alpha x_{i}^{\gamma}$ is approximated by choosing $\gamma$ close to one, that is, by approximating a linear prize function.

Note that decreasing prize functions $(\gamma<0)$ lead to lower expected highest effort levels than increasing price functions $(\gamma \in(0,1))$. This is worth pointing out, because Cohen et al. (2008) have shown that decreasing prize functions can play an effort-enhancing role when there is uncertainty about player types (See Section 2). However, even in their case, this requires the number of players to be sufficiently large.

\subsection{Beyond Interval Equilibria}

DEP is a convenient property of prize functions, but it is not always plausible. For instance, in patent races, extremely low efforts will typically not generate a positive prize. Therefore, we now show that, when DEP is violated, more general equilibria may exist.

\subsubsection{Equilibrium Characterization}

We will construct non-degenerate intervals $\left[H^{1}, J^{1}\right],\left[H^{2}, J^{2}\right], \ldots,\left[H^{K}, J^{K}\right]$ such that $H^{1}=H_{S}^{*}, J^{1}=T$ and $H^{k}>J^{k+1}$ so that one of the following two cases arises: (i) The MSE has support $\mathcal{H}^{k} \equiv\left[H^{1}, J^{1}\right] \cup\left[H^{2}, J^{2}\right] \cup \ldots \cup\left[H^{K}, J^{K}\right]$, where $H^{K}=0$ (Figure 3); (ii) the MSE has support $\{0\} \cup \mathcal{H}^{k}$ (not depicted). The sequences are constructed so that the restriction of $\frac{a\left(x_{i}\right)}{x_{i}}$ to $\mathcal{H}^{k}$ is monotone decreasing. We use the following mild non-linearity restriction.

(C4) $\frac{a\left(x_{i}\right)}{x_{i}}$ is non-constant on any open interval in $[0, T]$.

Specifically, we use the following recursive definition. 


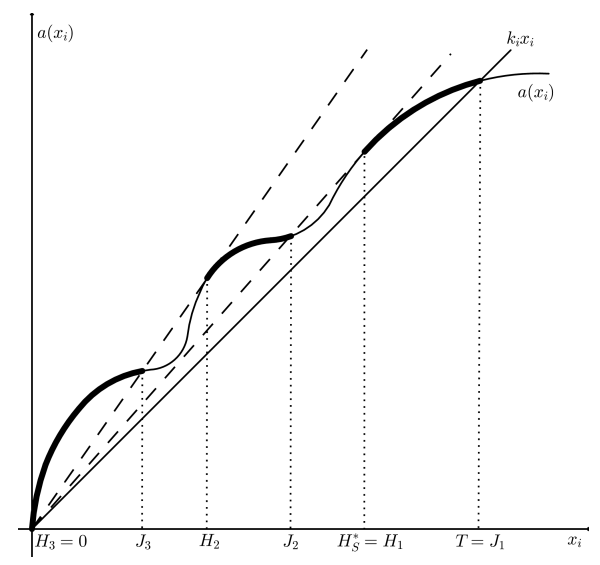

Figure 3: Equilibria with Non-Connected Support

Definition 7 Suppose $C 4$ holds. Let $J^{1}=T$ and $H^{1}=H_{S}^{*}$. Moreover, for all $k$ where the corresponding quantities are well-defined, let

$$
\begin{aligned}
& J^{k}=\max \left\{\begin{array}{l|l}
x_{i}<H^{k-1} & \begin{array}{c}
\frac{a\left(x_{i}\right)}{x_{i}}=\frac{a\left(H^{k-1}\right)}{H^{k-1}} \text { and } \exists \varepsilon>0 \text { such that } \\
\frac{a\left(x_{i}\right)}{x_{i}} \text { is strictly increasing on }\left(x_{i}-\varepsilon, x_{i}\right)
\end{array}
\end{array}\right\} \\
& H^{k}=\max \left\{x_{i}<J^{k} \mid \frac{a\left(x_{i}\right)}{x_{i}} \text { is strictly decreasing on }\left[x_{i}, J^{k}\right]\right\}
\end{aligned}
$$

We impose the following very weak restriction on $a\left(x_{i}\right)$.

(C5) $\frac{a\left(x_{i}\right)}{x_{i}}$ has finitely many local maxima on $[0, T]$.

Proposition 5 Suppose SYM, (C4) and (C5) hold

(i) If there exists $\varepsilon>0$ such that $\frac{a\left(\widetilde{x}_{i}\right)}{\widetilde{x}_{i}}>\max _{x_{i}>\widetilde{x}_{i}} \frac{a\left(x_{i}\right)}{x_{i}} \forall \widetilde{x}_{i} \in(0, \varepsilon]$, a MSE without atoms exists for which $\mathcal{S}=\mathcal{H}^{K}$ and $\left[F\left(x_{i}\right)\right]^{n-1} a\left(x_{i}\right)=x_{i}$ on $\mathcal{H}^{K}$. (ii)If $\max _{x_{i} \in(0, T)} \frac{a\left(x_{i}\right)}{x_{i}}$ exists, a MSE exists for which $\mathcal{S}=\{0\} \cup \mathcal{H}^{K}$. There is an atom at 0 with size $\left(\frac{H^{K}}{a\left(H^{K}\right)}\right)^{\frac{1}{n-1}}$. Also, $\left[F\left(x_{i}\right)\right]^{n-1} a\left(x_{i}\right)=x_{i}$ on $\mathcal{H}^{K}$.

The condition in (i) generalizes DEP: It does not require that $\frac{a\left(x_{i}\right)}{x_{i}}$ is decreasing globally, but nevertheless the highest values are attained near zero. In this case, the support still contains a non-degenerate interval including zero. When (ii) applies, low positive values (below $H^{k}>0$ ) are not attained.

We also have the following uniqueness result: 
Proposition 6 Suppose SYM, (C4) and(C5) hold. There exist no other equilibria than those described in Proposition 5.

\subsubsection{Implementation}

We restrict implementation results to the particularly interesting case of hurdle equilibria, which have the potentially attractive property that small positive efforts are not played. As argued repeatedly, it may be useful to have prize functions that generate effort distributions without small positive efforts. We first show that Proposition 5 implies a condition for existence of hurdle equilibria, which is, for instance, satisfied by $a^{5}$ in Figure 1:

Corollary 4 If $H_{S}^{*}>0$ is an interior global maximum of $\frac{a\left(x_{i}\right)}{x_{i}}$ on $[0, T]$, a hurdle equilibrium exists. The support is $\mathcal{S}=\{0\} \cup\left[H_{S}^{*}, T\right]$ and

$$
\begin{gathered}
{\left[F\left(x_{i}\right)\right]^{n-1}=\frac{x_{i}}{a\left(x_{i}\right)} \text { for } H_{S}^{*} \leq x_{i} \leq T} \\
\text { there is an atom at } 0 \text { with mass }\left(\frac{H_{S}^{*}}{a\left(H_{S}^{*}\right)}\right)^{\frac{1}{n-1}} .
\end{gathered}
$$

Corollary 4 leads to an implementation result for such distributions.

Proposition 7 Suppose SYM holds. Consider a strictly increasing CDF F with a density $f$ such that $\left\{x_{i} \mid f\left(x_{i}\right)>0\right\}=[0, C]$ for some $C>0$. For $H_{S} \in(0, C)$, let

$$
F^{H_{S}}\left(x_{i}\right)=\left\{\begin{array}{cll}
F\left(x_{i}\right) & \text { for } & x_{i}>H_{S} \\
F\left(H_{S}\right) & \text { for } & x_{i} \leq H_{S}
\end{array} .\right.
$$

Then, there exists an AEP for which the unique MSE is the hurdle equilibrium described in Corollary 4 with hurdle $H_{S}$.

The simplest way to construct the required prize function is by choosing $a^{F^{H_{S}}}\left(x_{i}\right)=0$ for $x_{i} \leq H_{S}$ and $a^{F^{H_{S}}}\left(x_{i}\right)=\frac{x_{i}}{\left(F\left(x_{i}\right)\right)^{n-1}}$ for $x_{i} \in\left(H_{S}, T\right]$; above $T, a^{F^{H_{S}}}$ can be extended by any function that satisfies $a^{F^{H_{S}}}\left(x_{i}\right) \leq x_{i}$. Implementation of a CDF as a hurdle equilibrium is unique only on the interval $\left[H_{S}, T\right]$. For values below the hurdle, any sufficiently kinked prize function guarantees that downward deviations from the hurdle are not attractive. 


\subsubsection{Optimality}

As argued above, it appears plausible that hurdle equilibria induce high expected highest efforts. To this end, we reconsider the class of power functions $\alpha x_{i}^{\gamma}$. We now fix $\gamma$ at specific values and consider hurdles that we allow to vary. Specifically, we write $H_{S}=h T$ for the threshold $T=\alpha^{\frac{1}{1-\gamma}}$ and $h \in(0,1)$. Using an analogous procedure as in Section 4.3 , we then consider parameter choices $h \in[0,1), \gamma \in[0,1)$ that yield the same expected average effort. It turns out that these level curves are upward sloping: If the overall level of prizes $\alpha$ is reduced, a reduction of the hurdle parameter $h$ is necessary to keep average efforts at the same level. Figure 4 depicts the ratio of expected highest to average efforts as a function of $h$ when expected average efforts are fixed at one. Thus, a simultaneous increase in the prize and the hurdle that keeps expected average efforts fixed increases expected highest efforts.

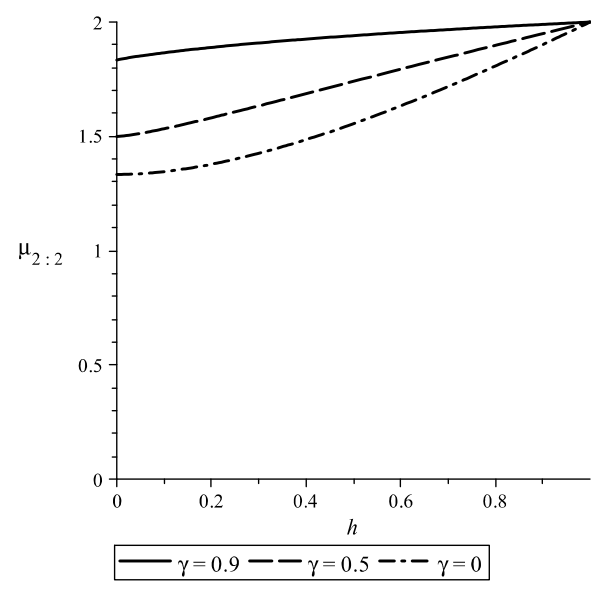

Figure 4: Expected highest effort with hurdle equilibria

\section{$5 \quad$ Asymmetric cost functions}

We now consider asymmetric costs functions. For simplicity, we use a more restrictive version of $\mathrm{A} 2$ from now on.

Assumption A2': $a\left(x_{i}\right)$ is (i) continuous, and (ii) continuously differentiable on $(0, \infty)$. 
We first deal with the characterization of the MSE and with implementation. As in Section 4.3, we then use a rich class of parameterized examples to show which prize functions yield high expected highest efforts.

\subsection{Characterization of MSE}

We confine ourselves to the two-player case. We suppose that $k_{1} \equiv k<1=$ $k_{2}$. Pure-strategy equilibria may exist for large cost asymmetries.

Proposition 8 If $n=2$ and $\arg \max _{x_{1} \in\left[0, r_{1}\right]} a\left(x_{1}\right)-k x_{1} \in\left[r_{2}, r_{1}\right]$, the AEP has a pure-strategy equilibrium $\left(x_{1}^{*}, x_{2}^{*}\right)$ with $x_{2}^{*}=0$.

In particular, a PSE exists if $a\left(x_{i}\right)$ is strictly concave and $a^{\prime}(T)>k$. From now on, we therefore assume that asymmetries are less pronounced, so that the following assumption holds together with A1, A2' and A3.

Assumption A6: $a^{\prime}(T)-k<0$

We give conditions under which we can characterize MSE. C1' replaces C1 with the condition from Siegel (2010). ${ }^{20}$

$\left(\mathrm{C} 1^{\prime}\right) a(0)>0$ and $a\left(x_{i}\right)-k x_{i}$ is strictly decreasing.

The alternative condition $\mathrm{C} 2$ is replaced as follows:

(C2') $a\left(x_{i}\right)$ is strictly concave.

Recall that $\mathrm{C} 2$ does not require concavity. However, contrary to C2, C2' does not restrict behavior of $a\left(x_{i}\right)$ near zero.

Definition 8 In the case of asymmetric cost functions, the effort hurdle, $H_{A}^{*}$, is given by $\min \left\{x_{i} \geq 0 \mid k a\left(x_{i}\right)-a^{\prime}\left(x_{i}\right)\left(w_{1}+k x_{i}\right) \geq 0\right\}$.

This generalizes Definition 6 , as $w_{1}=0$ for symmetric cost functions.

\footnotetext{
${ }^{20}$ To repeat, however, the framework of Siegel is more general in other dimensions.
} 
Proposition 9 (a) If $C 1^{\prime}$ or $C 2$ ' holds, an $M S E\left(F_{1}^{*}, F_{2}^{*}\right)$ exists such that: (i) $F_{1}^{*}$ has support $\mathcal{S}_{1}=\left[H_{A}^{*}, T\right]$ and

$$
F_{1}^{*}\left(x_{1}\right)=\frac{x_{1}}{a\left(x_{1}\right)} \text { for } H_{A}^{*} \leq x_{1} \leq T .
$$

(ii) $F_{2}^{*}$ has support $\mathcal{S}_{2}=\{0\} \cup\left[H_{A}^{*}, T\right]$ and

$$
F_{2}^{*}\left(x_{2}\right)=\left\{\begin{array}{ccc}
\frac{w_{1}+k H_{A}^{*}}{a\left(H_{A}^{*}\right)} & \text { for } & x_{2}=0 \\
\frac{w_{1}+k x_{2}}{a\left(x_{2}\right)} & \text { for } & H_{A}^{*} \leq x_{2} \leq T
\end{array} .\right.
$$

(b) $H_{A}^{*}=0$ if and only if

$$
a^{\prime}(0) w_{1}-k a(0) \leq 0
$$

(c) Expected payoffs are zero for player 2 and $w_{1}=(1-k) T$ for player 1 .

Several points are worth emphasizing. First, as in the symmetric case, the maximum of the support is the threshold for both players. Second, however, for strictly concave objective functions (C2'), the support is not necessarily the entire interval $[0, T]$. Instead, an asymmetric hurdle equilibrium can exist where players have the same minimal positive effort level $H_{A}^{*}$ in the support. (15) shows that this happens if asymmetries are large ( $k$ is small and $w_{1}$ is large) and zero efforts are unattractive $\left(a(0)\right.$ small and $a^{\prime}(0)$ high). Moreover, the high-cost player must have an atom at zero, whereas the low-cost player has an atom at $H_{A}^{*}$. Third, for the two-player case treated here, Proposition 9 goes beyond Theorem 1 of Siegel (2009) and Theorem 3 of Siegel (2010). Siegel requires that C1' holds. The proof of Part (b) of Proposition 9 shows that, in this case, the support is $[0, T]$ for both players, which is in line with Siegel's results. We show that the conclusion of Siegel can hold even if C1' is violated, but $\mathrm{C} 2$ ' holds instead. We also identify conditions under which the support is not $[0, T]$. Finally, part (c) of Proposition 9 holds much more generally, as Siegel (2012) has shown.

Moreover, all equilibria must be of the form just described.

Proposition 10 Suppose that C1' or C2' holds. Any MSE must be of the form described in Proposition 9. 
For C1', Proposition 10 is already shown by Siegel (2009, 2010). Our proof thus focuses on $\mathrm{C} 2$ '.

\subsection{Implementation}

Next, we present implementation results. Suppose given $H_{A}>0, C>H_{A}$ and CDFs $F_{1}$ and $F_{2}$ with supports $\left[H_{A}, C\right]$ and $\{0\} \cup\left[H_{A}, C\right]$, respectively. We ask under which conditions a prize function with $\operatorname{MSE}\left(F_{1}, F_{2}\right)$ exists. Propositions 9 and 10 imply that the equilibrium CDF $F_{2}$ must satisfy

$$
F_{2}\left(x_{2}\right)=\left\{\begin{array}{lll}
\left(\frac{(1-k) C}{x_{2}}+k\right) F_{1}\left(x_{1}\right) & \text { for } \quad H_{A}^{*} \leq x_{1}<C \\
\left(\frac{(1-k) C}{x_{2}}+k\right) F_{1}\left(H_{A}^{*}\right) & \text { for } \quad 0 \leq x_{1}<H_{A}^{*}
\end{array} .\right.
$$

Thus, once the CDF of the strong player is fixed, the CDF of the weak player is determined by (16). The only candidate prize function that can implement $F_{1}$ on $\left[H_{A}, C\right]$ is $a^{F_{1}}\left(x_{1}\right)=\frac{x_{1}}{F_{1}\left(x_{1}\right)}$. We start with implementation by a decreasing net prize function. By Proposition 9(b), this requires that the MSE has support $[0, C]$ for both players.

Proposition 11 Suppose a CDF $F_{1}$ has density $f_{1}$ and $\left\{x_{1} \mid f_{1}\left(x_{1}\right)>0\right\}=$ $[0, C]$ for $C>0$. Let $F_{2}$ be given by (16). Then $\left(F_{1}, F_{2}\right)$ can be implemented as the MSE of an AEP with a decreasing net prize function if and only if

$$
F_{1}\left(x_{1}\right)-x_{1} f_{1}\left(x_{1}\right)<k\left(F_{1}\left(x_{1}\right)\right)^{2} .
$$

(17) implies that the candidate net prize function is decreasing.

Next, we ask which CDFs can result as MSE for strictly concave $a\left(x_{i}\right)$.

Proposition 12 Suppose a $C D F F_{1}$ has differentiable density $f_{1}$ and $\exists C>$ $H_{A} \geq 0$ such that $\left\{x_{1} \mid f_{1}\left(x_{1}\right)>0\right\}=\left[H_{A}, C\right]$. If $F_{2}$ is given by $(16),\left(F_{1}, F_{2}\right)$ can be implemented as the MSE of an AEP with a strictly concave $a\left(x_{i}\right)$ if and only if

$$
\frac{f_{1}^{\prime}(x)}{f_{1}(x)}+\frac{2}{x}-2 \frac{f_{1}(x)}{F_{1}(x)}<0
$$

Compared to the symmetric case, several points are worth noting. We obtain analogous CDFs on $[0, C]$ as for the symmetric case as candidates for 
the effort distribution of the low-cost player. However, the lower end of the distribution is replaced by an atom. Moreover, the other player's CDF is completely determined by the choice of the first player's CDF.

\subsection{Optimality}

We analyze expected efforts for prize functions given by (8). We consider small asymmetry $(k=0.95)$ and large asymmetry $(k=0.85)$. Figure 5 describes the equilibrium CDF for $k=0.95$ and $\alpha=1$ and three different values of $\gamma,(0,0.5,0.8)$. We shall refer to these cases as the fixed prize case,
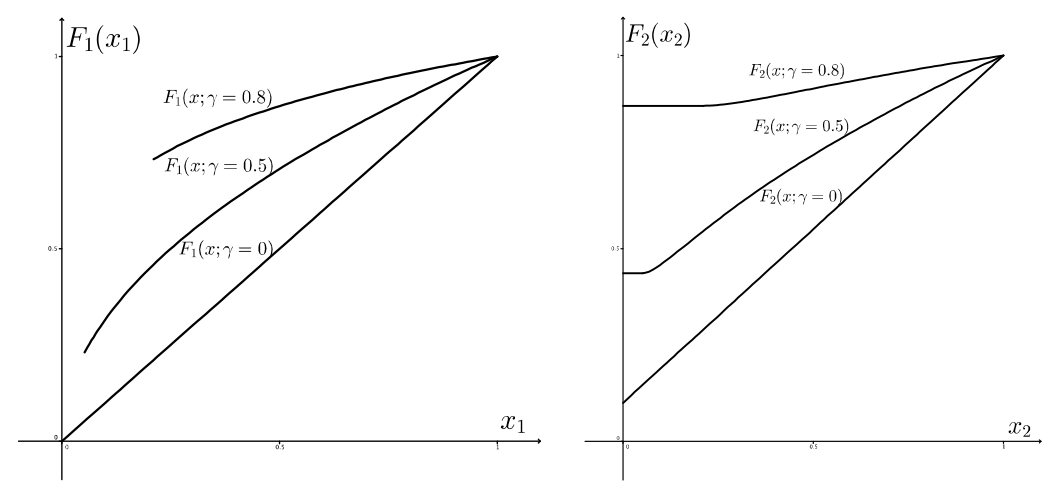

Figure 5: Equilibrium CDFs for player 1 (left) and 2 (right); $\alpha=1, k=0.95$ the intermediate case and the almost-linear case, respectively.

\subsubsection{Equilibrium structure}

In the fixed prize case, only the weak player has an atom at zero. As $\gamma$ increases, atoms for both players emerge and the support becomes smaller. For $k=0.85$ (not depicted), the supports are smaller than for $k=0.95$ and the atoms are larger; the MSE thus approaches an asymmetric PSE (which arises when $k \leq 1-\gamma$.) Figure A3 shows the density functions for both players.

\subsubsection{Comparative Statics}

Figure A4 displays individual efforts as a function of $\gamma$, with $\alpha$ fixed as 1 . The expected effort is higher for the strong player than for the weak player. 
An increase in asymmetry (reduction in $k$ ) implies higher expected effort for the strong player and lower expected effort for the weak player. Near the fixed prize case $(\gamma=0)$, the effort differences are small. Figure A5 displays expected average prizes, expected average efforts and expected highest efforts as a function of $\gamma$. Contrary to the symmetric case, the expected prizes and expected average efforts differ. Even though the weak player is exactly compensated for his efforts on expectation, the strong player's expected efforts differ from his expected prizes. His expected prizes and efforts are both above costs, and they are generally not identical.

Figure A6 shows that the winning probability of the more efficient player is far from 1 , in particular, for weak asymmetries $(k=0.95)$.

\subsubsection{Comparing efforts for prize-equivalent AEP}

Figures A7 and A8 show that the expected effort of player 1 (2) is increasing (decreasing) in $\gamma$ for fixed expected prize. As in the symmetric case, the expected highest effort is increasing in $\gamma$ (Figure 6) within a class of prize equivalent AEP. In particular, the expected highest effort is higher in the almost-linear case than in the fixed prize case. Figure 6 also shows that, contrary to the symmetric case, the expected average effort is non-monotone in $\gamma$ (with an interior minimum). As in the symmetric case, increasing $\gamma$ increases
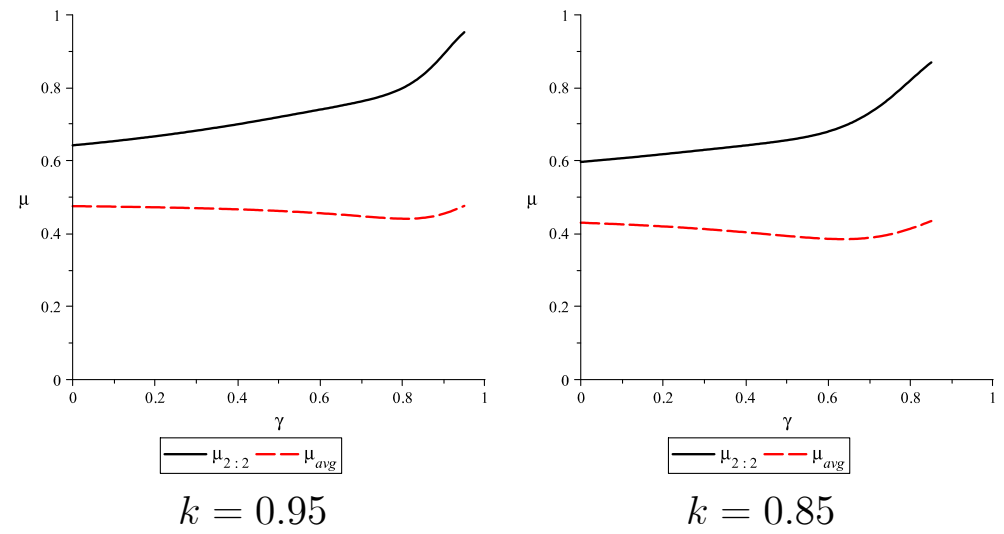

Figure 6: Expected highest effort and expected average effort for prizeequivalent APA, where the expected prize equals 1 
the expected highest effort. Contrary to the symmetric case, however, $\gamma$ has ambiguous effects on expected average efforts, because it increases the effort of the strong player and reduces the effort of the weak player.

\section{Applications}

This section shows how innovation races, promotion contests and beauty contests can be interpreted as AEP.

\subsection{Incentives for product innovations}

Consider the following set-up. Two symmetric firms spend R\&D costs $x_{i}$ to obtain a patent on a new product. The patent is awarded to the firm with the higher $x_{i} ; x_{i}$ is also positively related to product quality. Once one of the firms (say, firm 1) has obtained a patent, it is not possible without infringing on the patent to produce a substitute that prevents the successful firm from obtaining monopoly power. ${ }^{21}$ The R\&D effort is without value for the loser. Market demand is $D\left(p ; x_{1}\right)$, which is decreasing in $p$ and increasing in $x_{1}$. Let $p^{m}\left(x_{1}\right)$ be the monopoly price corresponding to quality $x_{1}$. For simplicity, marginal costs are constant and normalized to zero.

We can model the patent race between the two firms as an AEP with $a\left(x_{1}\right)=p^{m}\left(x_{1}\right) D\left(p^{m}\left(x_{1}\right) ; x_{1}\right)$, the monopoly profit of a firm with quality $x_{1}$. It is natural to assume that profits are increasing in quality. Moreover, in many cases, the profit function is concave in $x_{1}$. For instance, consider a linear demand function $D\left(p ; x_{1}\right)=\left(x_{1}\right)^{\gamma / 2}-p$, where $\gamma \in(0,1)$. Then, the positive demand effects of higher R\&D costs decline sufficiently fast that the monopoly profits $\frac{\left(x_{1}\right)^{\gamma}}{4}$ are concave under the parameter restriction. By Corollary 2, for parameters such that profits are increasing and concave in $x_{1}$, the resulting MSE has a decreasing density. Moreover, we can compare the expected quality in the market with a patent (which is the expected

\footnotetext{
${ }^{21}$ Alternatively, one might assume that there are no patents, but that the market can only sustain the better firm, which limit prices the competitor. Then, we obtain an all-pay auction with negative externalities, as treated experimentally by Darai et al. (2010) and Sacco and Schmutzler (2011).
} 
highest $\mathrm{R} \& \mathrm{D}$ effort) with the one that would have obtained in a race where the successful player obtains a fixed prize $(\gamma=0)$ which compensates him exactly for the expected average effort. Applying the arguments of Section 4.3 , the expected highest $\mathrm{R} \& \mathrm{D}$ effort is higher in the patent race than in the race where the fixed prize is given. Thus patents induce a higher expected highest effort for any given expected average effort than fixed prizes.

\subsection{Promotion contests}

Consider a promotion contest where two employees exert effort $y_{i}$ that is used to determine who is promoted to the higher level. The direct value of promotion is $A>0$. Suppose that the effort has additional benefits to the employee, because it is useful for his future career within the firm. For instance, the employee's knowledge about the organization might improve as he exerts more effort, making his life in the organization easier in the future. Suppose that these effects are potentially relevant if the employee is promoted and if he is not, but that their size is different in the two cases, given by $L\left(y_{i}\right)$ and $W\left(y_{i}\right)$, respectively. Net payoffs are therefore $L\left(y_{i}\right)-y_{i}$ for the loser and $A+W\left(y_{i}\right)-y_{i}$ for the winner.

Assume that $0 \leq L^{\prime}\left(y_{i}\right)<1$ and $0 \leq W^{\prime}\left(y_{i}\right)<1$. Then $\psi\left(y_{i}\right) \equiv y_{i}-$ $L\left(y_{i}\right)$ is a strictly increasing function capturing the effort cost net of benefits without promotion. Let $\phi\left(x_{i}\right) \equiv \psi^{-1}\left(x_{i}\right)$. Defining $x_{i} \equiv y_{i}-L\left(y_{i}\right)$, a loser who chooses $x_{i}$ earns a net prize of $-x_{i}$. A winner earns $A+W\left(y_{i}\right)-$ $y_{i}=A+W\left(\phi\left(x_{i}\right)\right)-L\left(\phi\left(x_{i}\right)\right)-x_{i}$. Thus, with the prize function $a\left(x_{i}\right) \equiv$ $\max \left(A+W\left(\phi\left(x_{i}\right)\right)-L\left(\phi\left(x_{i}\right)\right), 0\right)$, the game is an AEP if $A+W\left(\phi\left(x_{i}\right)\right)-$ $L\left(\phi\left(x_{i}\right)\right) \geq 0 \forall x_{i} \in \mathbb{R}^{+}$and A2 and A3 hold. A simple example where this is true is when there exist constants $\lambda, \omega \in(0,1)$ such that $L\left(y_{i}\right)=\lambda y_{i}$ and $W\left(y_{i}\right)=\omega y_{i}$. Then $\phi\left(x_{i}\right)=\frac{x_{i}}{1-\lambda}$ and, for $a\left(x_{i}\right) \equiv A+\frac{(\omega-\lambda) x_{i}}{1-\lambda}$. In particular, the prize function is decreasing if and only if $\omega<\lambda$, that is, if and only if effort has a stronger effect on the benefits in the current job than in the higher job.

Figure 7 shows the equilibrium distribution of $y_{i}$ in three different cases, in all of which $A=5$. The straight line in the middle depicts the uniform distribution of $y_{i}$ when $\omega=0.25$ and $\lambda=0.25$, so that the effort effects on 
$L$ and $W$ are the same and $W\left(\phi\left(x_{i}\right)\right)-L\left(\phi\left(x_{i}\right)\right) \equiv 0$ (fixed prize case). The convex CDF corresponds to $\omega=0$ and $\lambda=0.5$, so that exerting effort has positive effects only in the old job. The concave CDF corresponds to the case that $\omega=0.5$ and $\lambda=0$, so that exerting effort has positive effects only after promotion. Though the principal pays $A$ in each case, the expected efforts are highest in the latter case where the expected prize for the agents also consists of the expectation of $W\left(\phi\left(x_{i}\right)\right)-L\left(\phi\left(x_{i}\right)\right)>0$. Agents intrinsic concerns for their performance in the job can thus lead to higher or lower expected average efforts, depending on whether the efforts have stronger effects with or without promotion.

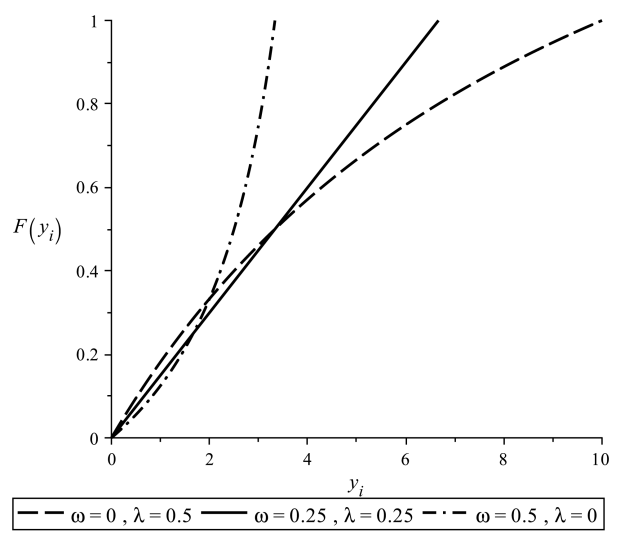

Figure 7: Effort distributions in the promotion game

\subsection{Beauty Contests}

Suppose a downstream firm (the client) wants to procure a task from one of two suppliers $i \in\{1,2\}$. These firms simultaneously choose costly and observable efforts $x_{i} \in \mathbb{R}^{+}$to prepare this project. Suppose the expected value of the project for the principal is an increasing function $V\left(x_{i}\right)$ of effort and that the party who is awarded the contract receives a fixed and commonly known prize $A>0 .{ }^{22}$ The client awards the task to the supplier who exerted the higher effort. After that, the supplier carries out the task at a cost of

\footnotetext{
${ }^{22}$ The analysis can be easily modified to account for the case that parties who exerted higher effort receive a higher payment.
} 
$C\left(x_{i}\right)$. This function can be decreasing or increasing. A decreasing function makes sense when high effort results in good preparation of the project, which makes it simpler to carry out. An increasing function is plausible when convincing the principal requires coming up with a project that is costly to finance ex post. In such a context, the logic of the previous subsection applies: When the interests of the client and the suppliers are aligned, that is, efforts reduce future costs of carrying out the project, the prize function is increasing in effort, conversely when efforts make the project more costly to carry out in the future.

\section{Conclusion}

Many economic institutions have features of all-pay auctions with endogenous prizes. This paper has shown that very general distributions can be obtained as mixed-strategy equilibria of all-pay auctions with endogenous prizes, at least in symmetric settings. It also shows which incentive systems yield high expected highest effort, while avoiding excessive effort of losers. Compared to all-pay auctions with fixed prizes, all-pay auctions with endogenous prizes generally do better in this respect, in particular, when prize functions are approximately linear. When the prize functions have hurdles below which there is no positive prize, this leads to further improvements.

The companion work of Jönsson (2013) analyzes symmetric AEP experimentally. Though the behavior of subjects differs substantially from the prediction of Proposition 1, prelimary results suggest that the comparative statics insights of Section 4.3 hold: Modifying an AEP in such a way that expected average efforts are fixed, but prizes become more sensitive to efforts yields higher expected efforts.

The paper can be extended in various directions. Richer objective functions of principals might be studied: Principals might be interested in other goals than maximizing expected average or highest effort. For instance, they might care about the minimal efforts, or they might want to secure that all players choose efforts close to some target level. And even if they care about the expected highest effort, they may be risk averse, which might lead to very different optimal incentive systems than those described above. Never- 
theless, the techniques developed in this paper would appear to be a useful step in deriving the appropriate systems for such alternative targets: The implementation results at least show what kind of behavior can be induced with suitable AEP.

\section{References}

Balakrishnan, N. (2007), "Permanents, Order Statistics, Outliers, and Robustness", Revista Matematica Complutense 20, no.1, 7-107.

Baye, M.R., Kovenock, D., and de Vries C.G. (2010), "Contests with RankOrder Spillovers", Economic Theory 51, 315-350.

Chowdhury, S., "The All-Pay Auction with Non-Monotonic Payoff", Working Paper, University of East Anglia.

Chung, T.Y. (1996), "Rent-Seeking Contest when the Prize Increases with Aggregate Efforts", Public Choice, 87, 55-66.

Cohen, C., Kaplan, T.R., and Sela, A. (2008), "Optimal Rewards in Contests", RAND Journal of Economics, 39, 434-451.

Darai, D., Sacco, D., and Schmutzler, A. (2010), "Competition and Innovation: An Experimental Investigation", Experimental Economics, 13, 439-460.

Jönsson, S. (2013), "All-Pay Auctions with Negative Externalities", Work in Progress, University of Zurich.

Kaplan, T.R., Luski, I., Sela, A., and Wettstein, D. (2002), "All-pay Auctions with Variable Rewards", Journal of Industrial Economics, L, 417430 .

Kaplan, T.R., Luski, I. and Wettstein, D. (2003), "Innovative Activity and Sunk Cost", International Journal of Industrial Organization, 21, 1111-1133.

Konrad, K.A. (2009), "Strategy and dynamics in contests", Oxford University Press.

Moldovanu, B., and Sela, A. (2001), "The Optimal Allocation of Prizes in Contests", The American Economic Review, 91, 542-558

Osborne, M. (2004), "An Introduction to Game Theory", Oxford University Press. 
Sacco, D. and Schmutzler, A. (2008), "All-Pay Auctions with Negative Prize Externalities: Theory and Experimental Evidence", Working paper series / Socioeconomic Institute No. 806.

Skaperdas, S. (1992) "Cooperation, Conflict, and Power in the Absence of Property Rights", American Economic Review, 82, 720-739.

Siegel, R. (2009), "All-Pay Contests", Econometrica, 77, 71-92.

Siegel, R. (2010), "Asymmetric Contests with Conditional Investments", American Economic Review, 100, 2230-2260.

Siegel, R. (2012), "Contests with Productive Efforts", Working Paper, Northwestern University. 


\section{APPENDIX}

\subsection{Appendix 1: Proofs}

\subsubsection{Proof of Proposition 1}

We appeal to the standard characterization result for MSE with continuous action spaces (adapted from Osborne 2004, Proposition 142.2).

Lemma $1 F$ is a MSE if and only if (i) there is no action which, given the opponents' behavior, yields higher expected payoff than $F$ and (ii) $F$ assigns zero probability to the set of actions for which, given the opponents' behavior, the expected payoff is less than her expected equilibrium payoff.

We now prove Proposition 1. DEP implies that $F\left(x_{i}\right)$ as in $(2)$ is strictly increasing on $(0, T]$. Moreover $F(T)=1$. If $a(0)>0(\mathrm{C} 1)$ or $a^{\prime}(0)=\infty$ (C2), then $\lim _{x_{i} \rightarrow 0} F\left(x_{i}\right)=0$ and $F$ is a CDF without atoms, with support $[0, T]$. If neither $\mathrm{C} 1$ nor $\mathrm{C} 2$ holds, but C3 does, then $\lim _{x_{i} \rightarrow 0}\left(F\left(x_{i}\right)\right)^{n-1}=$ $\frac{1}{\lim _{x_{i} \rightarrow 0} a\left(x_{i}\right)}>0$. Hence $\lim _{x_{i} \rightarrow 0} F\left(x_{i}\right)>0$ and $F$ is a CDF with support $[0, T]$ and an atom at zero with mass $\lim _{x_{i} \rightarrow 0} F\left(x_{i}\right)$. (2) implies that expected payoffs are zero on $[0, T]$. Because $a\left(x_{i}\right)<x_{i}$ for all $x_{i}>T, F$ corresponds to a MSE by Lemma 1 .

\subsubsection{Proof of Proposition 2}

We first prove several lemmas.

Lemma 2 If $S Y M$ holds, any symmetric MSE must satisfy (i) or (ii):

(i) It has no atoms, and $M \equiv \min \mathcal{S}=0$.

(ii) It has an atom at 0 with mass denoted as $F(0)$.

Case (ii) requires that $a(0)=0$ and $q_{n} a^{\prime}(0) \leq 1$ where $q_{n}=(F(0))^{n}$.

Proof. The proof has three steps:

Step 1: $M \equiv \min \mathcal{S}=0$ for every symmetric MSE without atoms.

Step 2: A symmetric MSE with an atom at $\widetilde{x}>0$ does not exist.

Step 3: A symmetric MSE with an atom at 0 requires $a(0)>0$ or $q_{n} a^{\prime}(0)>$ 1. 
Step 1: Suppose $M>0$. For any sequence $x_{n} \rightarrow M$, the probability of winning approaches zero. By continuity, $\lim _{x_{n} \rightarrow M} a\left(x_{n}\right)=a(M)<\infty$. The expected net payoff thus falls below zero as $x_{n} \rightarrow M$, so that $M \notin \mathcal{S}$.

Step 2: Suppose $\widetilde{x}>0$ is an atom. This requires $a(\widetilde{x}) \geq \widetilde{x}$. In the proposed MSE, for each player and each $m \in\{1, \ldots, n\}$, there is a probability $q_{m}>0$ that he is among the $m$ players with the highest effort. The expected net gain for a player from shifting the atom to the right by $\varepsilon$ is approximately $\sum_{m=2}^{n} q_{m} \frac{m-1}{m} a(\widetilde{x})+\sum_{m=1}^{n} \varepsilon q_{m} a^{\prime}(\widetilde{x})-\varepsilon$, which is positive if $a(\widetilde{x})>0$ and $\varepsilon$ is sufficiently small. Thus, shifting the atom to the right by some sufficiently small $\varepsilon$ increases expected payoffs, a contradiction.

Step 3: Suppose there is an atom at 0. Then in the proposed MSE, all players tie with some probability $q_{n}>0$. The expected net gain from shifting the atom to the right by $\varepsilon$ is approximately $q_{n} \frac{n-1}{n} a(0)+\varepsilon q_{n} a^{\prime}(0)-\varepsilon$, which is positive for sufficiently small $\varepsilon$ if $a(0)>0$ or $q_{n} a^{\prime}(0)>1$. Thus, unless $a(0)=0$ and $q_{n} a^{\prime}(0) \leq 1$, shifting the atom to the right by some sufficiently small $\varepsilon$ increases expected payoffs, a contradiction.

Lemma 3 If $S Y M$ holds, then $F\left(x_{i}\right)^{n-1} a\left(x_{i}\right)=x_{i}$ for all $x_{i} \in \mathcal{S} .^{23}$

Proof. By Lemma 2, for every symmetric MSE and all $\varepsilon>0$, there exist $x_{i}<\varepsilon$ such that $x_{i}$ is played. Also, there is no atom at 0 unless $a(0)=0$. Thus, expected payoffs approach zero as $x_{i}$ does. By Lemma 1 (i), there exists no $x_{i} \in X_{i}$ for which $F\left(x_{i}\right)^{n-1} a\left(x_{i}\right)-x_{i}>0$. Moreover, as $0 \in \mathcal{S}, F$ assigns zero probability to the set of $x_{i}$ for which $F\left(x_{i}\right)^{n-1} a\left(x_{i}\right)-x_{i}<0$ by Lemma 1(ii). Right continuity of $F$ and $a$ thus imply $F\left(x_{i}\right)^{n-1} a\left(x_{i}\right)-x_{i}=0$ $\forall x_{i} \in \mathcal{S}$.

Lemma 4 If $S Y M$ holds, $x_{L}, x_{H} \in \mathcal{S}$ and $0<x_{L}<x_{H}, \frac{a\left(x_{H}\right)}{x_{H}} \leq \frac{a\left(x_{L}\right)}{x_{L}}$.

Proof. (2) implies $\frac{a\left(x_{L}\right)}{x_{L}}=\frac{1}{\left[F\left(x_{L}\right)\right]^{n-1}}$ and $\frac{a\left(x_{H}\right)}{x_{H}}=\frac{1}{\left[F\left(x_{H}\right)\right]^{n-1}}$. Monotonicity of $F$ implies $\frac{a\left(x_{H}\right)}{x_{H}} \leq \frac{a\left(x_{L}\right)}{x_{L}}$.

Lemma 5 If $S Y M$ and DEP hold, there can be no $x^{L}<x^{H}$ such that $x^{L} \in \mathcal{S}$, $x^{H} \in \mathcal{S}, x^{L}>0$ and $\left(x^{L}, x^{H}\right) \cap \mathcal{S}=\varnothing$.

\footnotetext{
${ }^{23}$ For a similar, which is applicable if, e.g., C1 holds, see Siegel 2009, Corollary 3.
} 
Proof. There are no atoms at $x^{H}$ by Lemma 2. $\left(x^{L}, x^{H}\right) \cap \mathcal{S}=\varnothing$ would imply $F\left(x^{L}\right)=F\left(x^{H}\right)$. Thus, by Lemma $3, \frac{a\left(x^{H}\right)}{x^{H}}=\frac{a\left(x^{L}\right)}{x^{L}}$, violating DEP.

Lemma 6 If $S Y M$ and DEP hold, $\mathcal{S}=\{0\} \cup[L, T]$ for some $L \geq 0$.

Proof. By Lemma $2, \min \mathcal{S}=0$. By A3(i), $\mathcal{S} \subset[0, T]$. Next, for all $\varepsilon>0$, $\mathcal{S} \cap(T-\varepsilon, T] \neq \varnothing$, because there exists a left neighborhood of $T$ such that $a\left(x_{i}\right)-x_{i}>0$ in the entire neighborhood by A3(ii). Hence, $\max \mathcal{S}<T$ would imply that a player obtains a positive profit by choosing $x_{i} \in(\max \mathcal{S}, T)$. By Lemma $5, \mathcal{S} \cap(0, \infty)$ is an interval.

Lemma 7 If $S Y M$ holds, $\left[H_{S}^{*}, T\right] \subset \mathcal{S}$.

Proof. Suppose that $\left[H_{S}^{*}, T\right] \varsubsetneqq \mathcal{S}$. Arguments in the proof of Lemma 6 that do not depend on DEP show that $T \in \mathcal{S}$. Thus, there exists an $L \in$ $\left(H_{S}^{*}, T\right] \cap \mathcal{S}$ and $\varepsilon>0$ such that $(L-\varepsilon, L) \cap \mathcal{S}=\varnothing$. Choose $x_{i} \in(L-\varepsilon, L)$. The mass of players choosing $x_{i} \leq L$ is $\left(\frac{L}{a(L)}\right)^{\frac{1}{n-1}}$ and there is no atom at $L$ by Lemma 2. A player who deviates from $L$ to $x_{i}$ earns expected net payoffs $\frac{L}{a(L)} a\left(x_{i}\right)-x_{i}$. This is positive if $\frac{a\left(x_{i}\right)}{x_{i}}>\frac{a(L)}{L}$, which is true for $L>x_{i}>H_{S}^{*}$, a contradiction. Thus, $L=H_{S}^{*}$.

We now prove Proposition 2.

(i) From Lemmas 6 and Lemma 7, if SYM and DEP hold, $\mathcal{S}=\{0\} \cup$ $\left[H_{S}^{*}, T\right]$. Thus, $F\left(x_{i}\right)^{n-1} a\left(x_{i}\right)=x_{i}$ on $\left[H_{S}^{*}, T\right]$ by Lemma 3. DEP implies $H_{S}^{*}=0$. Thus the MSE is as described in Proposition 1.

(ii) Suppose DEP does not hold, that is, $H_{S}^{*}>0$. By Lemma $4,[0, T] \varsubsetneqq \mathcal{S}$. However, by Lemma $7,\left[H_{S}^{*}, T\right] \subset \mathcal{S}$. As $\mathcal{S} \subset[0, T]$, therefore there exists no $x_{i}>0$ such that $\mathcal{S}=\left[0, x_{i}\right]$.

\subsubsection{Proof of Proposition 3}

(i) Consider a CDF $F$ that admits a density $f$ such that $\left\{x_{i} \mid f\left(x_{i}\right)>0\right\}=$ $[0, C]$ for $C>0$. Then, $a^{F}$ as in (3) on $[0, C]$ is admissible: As to A2, differentiability holds on $(0, C]$. Continuity at 0 holds if and only if $\lim _{x_{i} \rightarrow 0} a^{F}\left(x_{i}\right)$ is finite, that is, (4) holds. In this case, $a^{F}$ is also right differentiable at 0. Further, $a^{F}$ can always be extended to the right in a continuously differentiable way such that $a^{F}\left(x_{i}\right)<x_{i}$ on $[C, \infty)$ and therefore A3(i) holds. A3(ii) 
holds because $\left(a^{F}\right)^{\prime}(C)<1$ for $f(C)>0$. It remains to be shown that $a^{F}$ satisfies C1 or C2 if and only if (5) holds. First, consider C1(ii) and C2(iii), that is, DEP. This requires

$$
\frac{F\left(x_{i}\right)-x_{i}(n-1) f\left(x_{i}\right)}{F\left(x_{i}\right)^{n}}<\frac{1}{F\left(x_{i}\right)^{(n-1)}} .
$$

(19) follows from $f\left(x_{i}\right)>0$. (5) is equivalent with the requirement that $\lim _{x_{i} \rightarrow 0} a^{F}\left(x_{i}\right)>0$ or $\lim _{x_{i} \rightarrow 0}\left(a^{F}\right)^{\prime}\left(x_{i}\right)=\infty$ if $\lim _{x_{i} \rightarrow 0} a^{F}\left(x_{i}\right)=0$, that is, with $\mathrm{C} 1(\mathrm{i})$ or $\mathrm{C} 2(\mathrm{i})$ and $\mathrm{C} 2(\mathrm{ii})$.

(ii) The preceding analysis shows that C3 holds in this case.

\subsubsection{Proof of Corollary 1}

For $a^{F}\left(x_{i}\right)=\frac{x_{i}}{\left[F\left(x_{i}\right)\right]^{n-1}},\left(a^{F}\right)^{\prime}\left(x_{i}\right)=\frac{\left[F\left(x_{i}\right)\right]-x_{i}(n-1) f\left(x_{i}\right)}{\left[F\left(x_{i}\right)\right]^{n}}>0$. (6) thus holds if and only if $a^{F}\left(x_{i}\right)$ is increasing on $(0, C]$. As $a^{F}(0)=\lim _{x_{i \rightarrow 0}} a^{F}\left(x_{i}\right), a^{F}\left(x_{i}\right)$ is increasing on $[0, C]$.

\subsubsection{Proof of Corollary 2}

Implementability follows immediately from Proposition 3: For $n=2$, (4) holds if $f\left(x_{i}\right)>0$ and (5) holds if $f\left(x_{i}\right)$ is bounded. Part (i) and (ii) of the Corollary are straightforward. (iii) is implied by (i) and (ii).

\subsubsection{Proof of Corollary 3}

$a^{F}\left(x_{i}\right)$ as in (3) violates (4) if $\lim _{x_{i} \rightarrow 0} f\left(x_{i}\right)<\infty$.

\subsubsection{Proof of Proposition 5}

(i) By (C4) and (C5), the sequences $H_{k}$ and $J_{k}$ are well-defined and stop after finitely many iterations. $F$ defines a distribution: It is increasing on each $\left[H^{k}, J^{k}\right]$, satifies $F\left(J^{k+1}\right)=F\left(H^{k}\right), F(0) \geq 0$ and $F\left(J^{1}\right)=F(T)=1$. All $x_{i} \in \mathcal{H}^{K}$ yield zero expected payoffs. For $x_{i} \notin \mathcal{H}^{K}$, expected payoffs are negative if $x_{i}>J^{1}=T$. If $x_{i} \in\left(J^{k+1}, H^{k}\right)$ for $k \in 1, \ldots, k-1$, then $\frac{a\left(x_{i}\right)}{x_{i}} \leq \frac{a\left(H^{k}\right)}{H^{k}}$. Expected payoffs $a\left(x_{i}\right) \frac{H^{k}}{a\left(H^{k}\right)}-x_{i}$ are therefore non-positive. (ii): analogous. 


\subsubsection{Proof of Proposition 6}

By Lemma 3, $\left[F\left(x_{i}\right)\right]^{n-1} a\left(x_{i}\right)=x_{i}$ on $\mathcal{S}$. Second, we show that $\mathcal{H}^{K} \subset$ $\mathcal{S}$. From Lemma $7,\left[H^{1}, J^{1}\right] \subset \mathcal{S}$. Next suppose $\left[H^{k}, J^{k}\right] \subset \mathcal{S}$, but $\exists x_{i}$ $\in\left[H^{k+1}, J^{k+1}\right] \notin \mathcal{S}:$ Clearly, for every $\widetilde{x}_{i} \in\left(H^{k+1}, J^{k}\right]$ and all $\varepsilon>0$ $\exists x_{i} \in\left(\widetilde{x}_{i}-\varepsilon, \widetilde{x}_{i}\right)$ such that $a\left(x_{i}\right) \frac{H^{k}}{a\left(H^{k}\right)}-x_{i}>0$, a contradiction. Thus, $\left[H^{k+1}, J^{k+1}\right] \subset \mathcal{S}$ and, by induction, $\mathcal{H}^{K} \subset \mathcal{S}$. Finally, we show that $\mathcal{S} \subset\{0\} \cup \mathcal{H}^{K}$. For $x_{i}>J^{1}=T$, expected payoffs are negative. Moreover, by Lemma 4 , if $x_{L} \in \mathcal{S}$ and $x_{H} \in \mathcal{S}, \frac{a\left(x_{H}\right)}{x_{H}} \leq \frac{a\left(x_{L}\right)}{x_{L}}$. Thus, as $\left[H^{k}, J^{k}\right] \subset \mathcal{S}$, $\left(J^{k+1}, H^{k}\right) \cap \mathcal{S}=\varnothing$. If the conditions of Proposition 5(i) hold, $H^{K}=0$ and thus $\mathcal{S} \subset\{0\} \cup \mathcal{H}^{K}=\mathcal{H}^{K}$. If the conditions of Proposition 5(ii) hold, $H^{K}>0$ and $F\left(H^{k}\right)=\frac{H^{k}}{a\left(H^{k}\right)}>0$. Hence, there is an atom at 0 or $H^{k}$. The latter possibility violates Lemma 2 .

\subsubsection{Proof of Corollary 4}

The assumptions imply that $K=1$ and $H^{1}=0$. Thus, Proposition 5 gives the result.

\subsubsection{Proof of Proposition 7}

Let $a^{F^{H}}\left(x_{i}\right)=\left\{\begin{array}{ccc}0 & \text { if } & x_{i} \in\left[0, H_{S}\right) \\ \left(\frac{x_{i}}{F\left(x_{i}\right)}\right)^{\frac{1}{n-1}} & \text { if } \quad x_{i} \geq H_{S}\end{array} . H_{S}\right.$ is the global maximum of $\frac{a^{F^{H} S}\left(x_{i}\right)}{x_{i}}$ on $(0, T]$, and it is interior. Corollary 4 yields the result.

\subsubsection{Proof of Proposition 8}

Let $x_{1}^{*}=\arg \max _{x_{1} \in\left[0, r_{1}\right]} a\left(x_{1}\right)-k x_{1}, x_{2}^{*}=0$. By construction, $x_{1}^{*}$ is a best response for player 1 . Because $x_{1}^{*}>r_{2}$, player 2 will not deviate to $x_{2} \geq x_{1}^{*}$. Deviation to $x_{2} \in\left(0, x_{1}^{*}\right)$ yields negative payoffs.

\subsubsection{Proof of Proposition 9}

We first prove a series of lemmas.

Lemma 8 (i) Player 2 earns expected payoffs of zero on $\{0\} \cup\left[H_{A}^{*}, T\right]$. (ii) Player 1 earns expected payoffs $w_{1}=(1-k) T$ on $\left[H_{A}^{*}, T\right]$. 
Proof. (i) Choosing $x_{2}=0$ yields expected payoffs of 0 if $a(0)=0$ or if $H_{A}^{*}>0$. If $a(0)>0$ and $H_{A}^{*}=0$, then $\lim _{x_{1} \rightarrow 0} F_{1}\left(x_{1}\right)=0$, so that expected payoffs of player 2 are still zero if he chooses 0 . (13) implies that net expected payoffs are zero for $x_{2}>0$ as well. (ii) By (14), player 1 obtains expected payoff $w_{1}$ for all $x_{1} \in\left[H_{A}^{*}, T\right]$.

Lemma 9 Deviations of player $i=1,2$ to $x_{i} \notin \mathcal{S}_{i}$ are non-profitable if

$$
H_{A}^{*}=0 \text { or } k a\left(H_{A}^{*}\right)-a^{\prime}\left(H_{A}^{*}\right)\left(w_{1}+k H_{A}^{*}\right) \leq 0 .
$$

Proof. By definition of $T$, deviations of player 2 to $x_{2}>T$ are nonprofitable. As C1' and C2' each imply $a^{\prime}\left(x_{1}\right)<k$ for $x_{1}>T$, player 1 does not benefit from deviating to $x_{1}>T$. Thus deviations to $x_{i} \notin \mathcal{S}_{i}$ are unprofitable if $H_{A}^{*}=0$ and, in particular, under C1'. If C2' holds and $H_{A}^{*}>0$, player 2 cannot deviate profitably to $x_{2} \in\left(0, H_{A}^{*}\right)$, as this would involve positive efforts without ever obtaining the prize. Player 1 cannot profitably deviate to $x_{1} \in\left(0, H_{A}^{*}\right)$ if $a^{\prime}\left(x_{1}\right) F_{2}(0)-k \geq 0 \forall x_{1} \in\left(0, H_{A}^{*}\right]$. As C2' requires concavity of $a\left(x_{i}\right)$, this holds if $a^{\prime}\left(H_{A}^{*}\right) F_{2}(0)-k \geq 0$. Inserting $F_{2}(0)$ gives $a^{\prime}\left(H_{A}^{*}\right) \frac{w_{1}+k H_{A}^{*}}{a\left(H_{A}^{*}\right)}-k \geq 0$, that is, $k a\left(H_{A}^{*}\right)-a^{\prime}\left(H_{A}^{*}\right)\left(w_{1}+k H_{A}^{*}\right) \leq 0$.

Lemma $10 F_{1}^{*}$ is a CDF. $F_{2}^{*}$ is a CDF if and only if

$$
k a\left(H_{A}^{*}\right)-a^{\prime}\left(H_{A}^{*}\right)\left(w_{1}+k H_{A}^{*}\right) \geq 0
$$

Proof. By definition of $T, F_{1}^{*}(T)=1$. By C1' or C2', $F_{1}^{*}\left(x_{1}\right)$ is increasing on $[0, T]$. Thus, (13) defines a CDF; it has an atom at $H_{A}^{*}$ with mass $F_{1}^{*}\left(H_{A}^{*}\right)=\frac{H_{A}^{*}}{a\left(H_{A}^{*}\right)}$. For $F_{2}^{*}$ to be a CDF, it has to be increasing, which requires

$$
k a\left(x_{2}\right)-a^{\prime}\left(x_{2}\right)\left(w_{1}+k x_{2}\right) \geq 0 \forall x_{2} \in\left[H_{A}^{*}, T\right] .
$$

C1' implies (22) because $a^{\prime}\left(x_{2}\right)<k$ and $w_{1}=a(T)-T<a\left(x_{2}\right)-x_{2}$. If C2' holds, the left-hand side of (22) is increasing in $x_{2}$. Thus, (22) holds on $\left[H_{A}^{*}, T\right]$ if and only if $k a\left(H_{A}^{*}\right)-a^{\prime}\left(H_{A}^{*}\right)\left(w_{1}+k H_{A}^{*}\right) \geq 0$. 


\section{Lemma 11 If}

$$
k a(0)-a^{\prime}(0) w_{1} \geq 0 .
$$

(20) and (21) both hold for $H_{A}^{*}=0$.

Proof. $H_{A}^{*}=0$ implies (20). (23) is (21) for $H_{A}^{*}=0$.

Lemma 12 If C2' holds and

$$
k a(0)-a^{\prime}(0) w_{1}<0,
$$

$H_{A}^{*} \in(0, T)$ is the only effort level that satisfies both (20) and (21).

Proof. For $x_{1}>0,(20)$ and (21) hold if and only if

$$
k a\left(x_{1}\right)-a^{\prime}\left(x_{1}\right)\left(w_{1}+k x_{1}\right)=0
$$

(25) holds for $x_{1}=H_{A}^{*}$ as $H_{A}^{*}>0$. C2' implies that the left hand side of (25) is increasing, so that the solution is unique.

We now derive Proposition 9. If C1' holds, $a(0)>a(T)-k T=w_{1}$ and $k>a^{\prime}(0)$. Thus (23) holds. Hence, by Lemma 11, (20) and (21) hold for $H_{A}^{*}=0$. Thus, Lemmas 9 and 10 imply that Part (a) of Proposition 9 holds. If C2' holds, Lemma 12 implies that $H_{A}^{*}>0$ satisfies (20) and (21); thus $F_{1}$ and $F_{2}$ are distributions by Lemma 10, and they correspond to an MSE by Lemma 9; so that Part (a) of the Proposition also holds in this case. Part (b) follows from the Definition of $H_{A}^{*}$. Part (c) follows from Lemma 8.

\subsubsection{Proof of Proposition 10}

As the result for C1' has been shown by Siegel (2009, 2010), we confine ourselves to C2'. The proof follows from Lemmas 13-22. With few exceptions, these results are so general that they do not require $\mathrm{C} 2$ ' (or $\mathrm{C} 1$ ').

Lemma $13 \mathcal{S}_{i} \subset[0, T]$ for any $M S E$ and $i=1,2$.

Proof. $x_{2}>T$ is not a best response for player 2 because $a\left(x_{2}\right)<x_{2}$. Thus $\mathcal{S}_{2} \subset[0, T]$. If player 1 chooses $x_{1}>T$, his net payoff is thus $a\left(x_{1}\right)-k x_{1}$. As $a^{\prime}\left(x_{i}\right)<k$ for all $x_{i} \geq T$, there exists an $\widetilde{x}_{1}$ in $\left(T, x_{1}\right)$ such that the net payoff is $a\left(\widetilde{x}_{1}\right)-k \widetilde{x}_{1}>a\left(x_{1}\right)-k x_{1}$. Thus $x_{1}>T$ is not a best response. 
Lemma 14 If player $i$ has an atom in $x^{*}$, then for $j \neq i$, there exists an $\varepsilon>0$ such that there are no best responses in $\left[x^{*}-\varepsilon, x^{*}\right]$.

Proof. Suppose player $i$ has an atom in $x^{*}$ with mass $p\left(x^{*}\right)$. Suppose for all $\varepsilon>0$ there exists a best response $x_{j} \leq x^{*}$ such that $\left|x_{j}-x^{*}\right|<\varepsilon$. By deviating to $x_{j}+\varepsilon$, player $j$ would increase his expected prize by at least approximately $p\left(x^{*}\right) \frac{a\left(x^{*}\right)}{2}$; his costs would increase by $\varepsilon$. As $\varepsilon \rightarrow 0$, the increase in the expected prize is higher than the increase in expected costs, so that $x_{j}$ is not a best response.

Lemma 15 In any $M S E, 0 \in \mathcal{S}_{2}$ and the expected net payoff of player 2 is zero, that is, $F_{1}\left(x_{2}\right) a\left(x_{2}\right)=x_{2}$ for all best responses $x_{2}$.

Proof. Player 2 obtains a payoff of at least zero by choosing 0 . Thus, $F_{1}\left(x_{2}\right) a\left(x_{2}\right) \geq x_{2}$ for any best response and, by continuity, for any $x_{2} \in \mathcal{S}_{2}$. To show that Player 2 does not obtain an expected payoff above zero, it suffices to show that there exists a best response $x_{2}$ for which he wins with probability arbitrarily close to zero. Let $\underline{x} \equiv \inf \mathcal{S}_{1} \cup \mathcal{S}_{2}$. First, suppose no player has an atom at $\underline{x}$. By definition of $\underline{x}$ one can find a sequence $x_{n}$ converging to $\underline{x}$ such that $x_{n}$ is a best response for at least one player $i$. As there is no atom at $\underline{x}$, the probability of winning and thus the expected payoff converges to zero as $x_{n} \rightarrow \underline{x}$. By Lemma 13, player 1 obtains a payoff of approximately $w_{1}$ by choosing $x_{1}$ just above $T$. Thus, $x_{n}$ cannot consist of best responses for player 1 . Hence, the $x_{n}$ are best responses of player 2 who therefore obtains a payoff of zero in the MSE. Second, suppose exactly one player has an atom at $\underline{x}$. Then this player obtains zero payoffs at $\underline{x}$; and it must therefore be player 2. Third, by Lemma 14, it is impossible that both players have atoms at $\underline{x}$. Finally, $\underline{x}=0$ and thus $\underline{x} \in \mathcal{S}_{2}$ : Because player 2 wins with probability zero, his net payoffs would be negative if $\underline{x}>0$.

Lemma $16 \max \mathcal{S}_{i}=T$ for $i=1,2$.

Proof. By Lemma 13, it suffices to show that $\max \mathcal{S}_{i} \geq T$. If $\max \mathcal{S}_{1}<T$, then by A3(ii), given the equilibrium strategy of player 1, player 2 could obtain positive payoffs by choosing $x_{2} \in\left(\max \mathcal{S}_{1}, T\right)$, contradicting Lemma 15. If $\max \mathcal{S}_{2}<T, a^{\prime}(T)-k<0$ implies that player 1 could profitably deviate downwards from $T$. Therefore $\max \mathcal{S}_{2}=T$. 
Lemma 17 If DEP holds, there can be no atom of player 2 at any $x^{*}>0$.

Proof. If player 2 has an atom at $x^{*}>0$, then by Lemma 14 there exists an $\varepsilon>0$ such that there are no best responses of player 1 in $\left(x^{*}-\varepsilon, x^{*}\right]=$ $\varnothing$. Thus, by choosing $x_{2} \in\left(x^{*}-\varepsilon, x^{*}\right)$, player 2 would obtain profits of $F_{1}\left(x^{*}\right) a\left(x_{2}\right)-x_{2}$. By Lemma 15, these profits are $\frac{x^{*}}{a\left(x^{*}\right)} a\left(x_{2}\right)-x_{2}$. Optimality of $x^{*}$ thus requires $\frac{x^{*}}{a\left(x^{*}\right)} a^{\prime}\left(x^{*}\right)>1$, violating DEP.

Lemma 18 The expected payoff of player 1 is $w_{1}$ in any MSE.

Proof. By Lemma 16, given the equilibrium strategy of player 2, player 1 can guarantee himself a payoff of arbitrarily close to $w_{1}>0$ with certainty by choosing an effort level just above $T$. By Lemma 17, player 2 cannot have an atom at $T$. Therefore, player 1 must obtain an expected payoff of exactly $w_{1}$ at $T$.

Lemma 19 Let $H_{1} \equiv \min \mathcal{S}_{1}$.Then $\left(0, H_{1}\right) \cap \mathcal{S}_{2}=\varnothing$.

Proof. Player 2's expected payoffs for $x_{2} \in\left(0, H_{1}\right)$ are negative.

Lemma 20 (i) If C2' holds, then $\nexists \underline{x}, \bar{x} \in \mathcal{S}_{1}$ with $\underline{x}<\bar{x}$ such that $(\underline{x}, \bar{x}) \cap$ $\mathcal{S}_{2}=\varnothing$.

(ii) If C2' holds, then $\nexists \underline{x}, \bar{x} \in \mathcal{S}_{2} \cap(0, \infty)$ with $\underline{x}<\bar{x}$ such that $(\underline{x}, \bar{x}) \cap \mathcal{S}_{1}=\varnothing$.

Proof. (i) Suppose $\exists \underline{x}, \bar{x} \in \mathcal{S}_{1}$ with $\underline{x}<\bar{x}$ such that $(\underline{x}, \bar{x}) \cap \mathcal{S}_{2}=$ $\varnothing$. If so, then, by choosing $x_{1} \in(\underline{x}, \bar{x})$, player 1 would obtain profits of $F_{2}(\underline{x}) a\left(x_{1}\right)-x_{1}$ and thus, using Lemma 17, $F_{2}(\bar{x}) a\left(x_{1}\right)-x_{1} . \bar{x} \in \mathcal{S}_{1}$ thus requires $F_{2}(\bar{x}) a^{\prime}(\bar{x})=F_{2}(\underline{x}) a^{\prime}(\bar{x}) \geq 1 . \underline{x} \in \mathcal{S}_{1}$ requires $F_{2}(\underline{x}) a^{\prime}(\underline{x}) \leq 1$. These two conditions together violate $\mathrm{C} 2$ '.

(ii) Suppose $\exists \underline{x}, \bar{x} \in \mathcal{S}_{2} \cap(0, \infty)$ with $\underline{x}<\bar{x}$ such that $(\underline{x}, \bar{x}) \cap \mathcal{S}_{1}=\varnothing$. Then $\underline{x} \notin \mathcal{S}_{1}$ is impossible. To see this, note that Player 2 has no atoms at any $x_{2}>0$ by Lemma 17. Thus $\lim _{\varepsilon \rightarrow 0} F_{2}(\underline{x}-\varepsilon)=F_{2}(\underline{x})$ and $\forall \varepsilon>0 \exists \delta \in$ $(0, \varepsilon)$ such that $F_{2}(\underline{x}-\delta)-F_{2}(\underline{x}-\varepsilon)>0$. If $\underline{x} \notin \mathcal{S}_{1}, \varepsilon$ can be chosen so that $F_{1}\left(x_{2}\right) a\left(x_{2}\right)=F_{1}(\underline{x}-\varepsilon) a\left(x_{2}\right)$ on $[\underline{x}-\varepsilon, \underline{x}-\delta]$. Thus $F_{1}(\underline{x}-\varepsilon) a^{\prime}\left(x_{2}\right) \equiv 1$ on this interval, violating $\mathrm{C} 2$ '. By analogous arguments, $\bar{x} \notin \mathcal{S}_{1}$ is impossible. Thus, $\underline{x}, \bar{x} \in \mathcal{S}_{1}$ and (i) shows that $(\underline{x}, \bar{x}) \cap \mathcal{S}_{2} \neq \varnothing$. As player 2 has no 
atom, this would require that there exists an open subinterval of $(\underline{x}, \bar{x})$ in $\mathcal{S}_{2}$. Because $(\underline{x}, \bar{x}) \cap \mathcal{S}_{1}=\varnothing$, this would imply that $F_{1}(\underline{x}) a\left(x_{2}\right)-x_{2}$ is constant on $(\underline{x}, \bar{x})$, which is incompatible with $\mathrm{C} 2$ '.

Lemma 21 If C2' holds, $\exists H_{A}>0$ such that $\mathcal{S}_{1}=\left[H_{A}, T\right]$ and $\mathcal{S}_{2}=\{0\}$ $\cup\left[H_{A}, T\right]$.

Proof. Let $H_{2}=\min \left(\mathcal{S}_{2} \cap(0, \infty)\right)$. By Lemma 19, $H_{1} \leq H_{2}$. We show that $H_{1}<H_{2}$ is impossible. First, suppose there exists $x_{1} \in\left(H_{1}, H_{2}\right) \cap \mathcal{S}_{1}$. This implies $F_{2}(0) a\left(H_{1}\right)-H_{1}=F_{2}(0) a\left(x_{1}\right)-x_{1}$, violating C2'. Second, suppose $\left(H_{1}, H_{2}\right) \cap \mathcal{S}_{1}=\varnothing$. Thus, $H_{1}$ must be an atom of player 1 . As $H_{2}$ is not an atom of player 2 by Lemma $17, F_{2}\left(H_{1}\right)=F_{2}\left(H_{2}\right)=F_{2}(0)$ and $H_{2} \in \mathcal{S}_{1}$. Thus, $F_{2}(0) a^{\prime}\left(H_{1}\right) \leq 1$ and $F_{2}(0) a^{\prime}\left(H_{2}\right) \geq 1$. These conditions together violate $\mathrm{C} 2$ '.

Thus $H_{1}=H_{2}$. Therefore, if $H_{1}=0$, then $H_{2}=0$ and, by Lemma 16 , $\{0, T\} \subset \mathcal{S}_{i}(i=1,2)$. Suppose $\mathcal{S}_{i} \varsubsetneqq[0, T]$. Then there exist $\bar{x}>\underline{x}>0$ such that $(\underline{x}, \bar{x}) \cap \mathcal{S}_{i}=\varnothing$. Hence, by Lemma $20, \underline{x} \notin \mathcal{S}_{j}$ or $\bar{x} \notin \mathcal{S}_{j}(j \neq i)$. Thus, there exists a subinterval of $(\underline{x}, \bar{x})$ which has empty intersection with $\mathcal{S}_{1}$ and $\mathcal{S}_{2}$. Choose the interval such that $\underline{x}$ is minimal and $\bar{x}$ is maximal. Then by Lemma 17, there can be no atom of player 2 in $\underline{x}$ or $\bar{x}$. Thus either there is an atom of player 1 at these effort levels or $\mathcal{S}_{2}$ must contain intervals of the form $(\underline{x}-\varepsilon, \underline{x})$ or $(\bar{x}, \bar{x}+\varepsilon)>0$. Because of $\mathrm{C}^{2}$, the latter possibility can only arise if $\underline{x} \in \mathcal{S}_{1}\left(\bar{x} \in \mathcal{S}_{1}\right)$. In any event, $\underline{x} \in \mathcal{S}_{1}, \bar{x} \in \mathcal{S}_{1}$ and $(\underline{x}, \bar{x}) \cap \mathcal{S}_{2}=\varnothing$, which is inconsistent with C2' by Lemma 20. If $H_{1}=H_{2} \equiv H_{A}>0$, analogous arguments show that $\mathcal{S}_{1}=\left[H_{A}, T\right]$ and $\mathcal{S}_{2} \cap(0, \infty)=\left[H_{A}, T\right]$ by Lemma 20.

Lemma 22 Suppose C2' holds and $H_{A} \neq H_{A}^{*}$. Then there can be no MSE with $\mathcal{S}_{1}=\left[H_{A}, T\right]$ and $\mathcal{S}_{2}=\{0\} \cup\left[H_{A}, T\right]$.

Proof. This follows from Lemma 12.

Proposition 10 now follows immediately: Lemmas 21 and 22 imply that $\mathcal{S}_{1}=\left[H_{A}^{*}, T\right]$ and $\mathcal{S}_{2}=\{0\} \cup\left[H_{A}^{*}, T\right]$. Lemmas 15 and 18 imply that the distribution must satisfy (13) and (14). 


\subsubsection{Proof of Proposition 11}

We first show that (17) is sufficient for implementation by monotone net prize functions. By Proposition 9, this requires that $a\left(x_{1}\right)$ satisfies A2 and A3 and C1'. As to A2, the candidate function $a\left(x_{1}\right)=\frac{x_{1}}{F_{1}\left(x_{1}\right)}$ is continuously differentiable for $x_{1}>0$. Continuity at 0 requires $\lim _{x_{1} \rightarrow 0} a\left(x_{1}\right)=\frac{1}{f_{1}(0)}<\infty$, which is equivalent with $f_{1}(0)>0$. A3 is clearly satisfied. C1' is equivalent with (17). Necessity follows because A2 is violated if (17) does not hold.

\subsubsection{Proof of Proposition 12}

We show that (18) is sufficient for implementation by a strictly concave price function. By Proposition 9, this is true if the candidate prize function satisfies A2 and A3 and C2'. A2 follows as in the proof of Proposition 11. A3 is clearly satisfied. C2' is equivalent with (18). 


\subsection{Appendix 4: Figures}

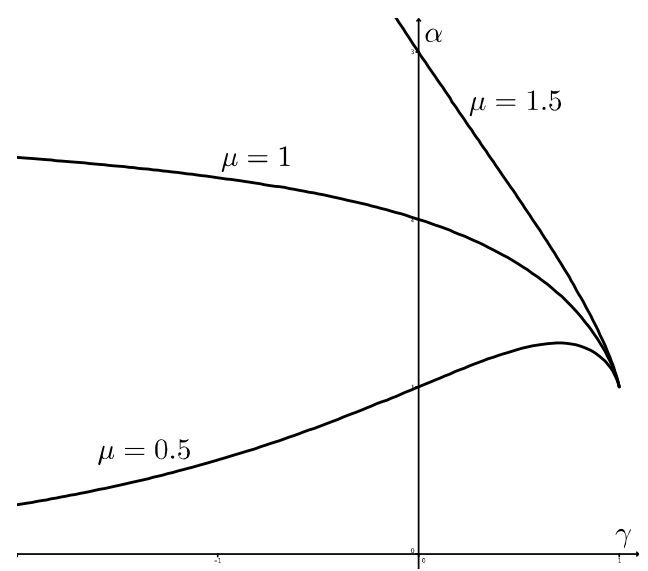

Figure A1: Prize-equivalent APA

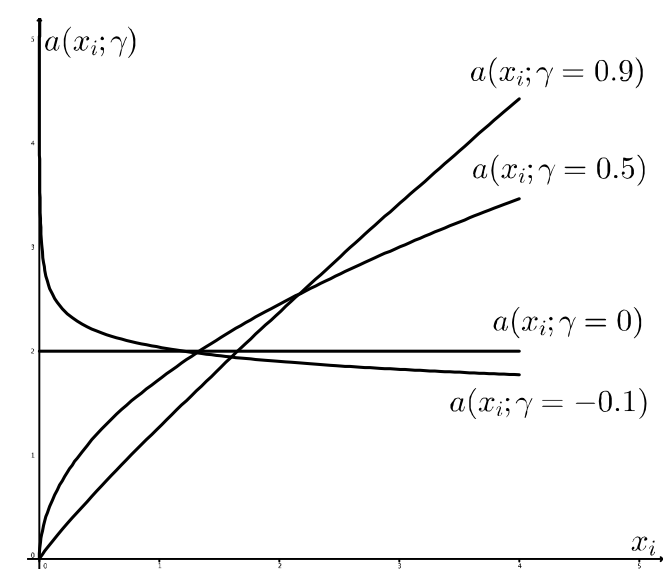

Figure A2: Prize functions corresponding to prize-equivalent APA 

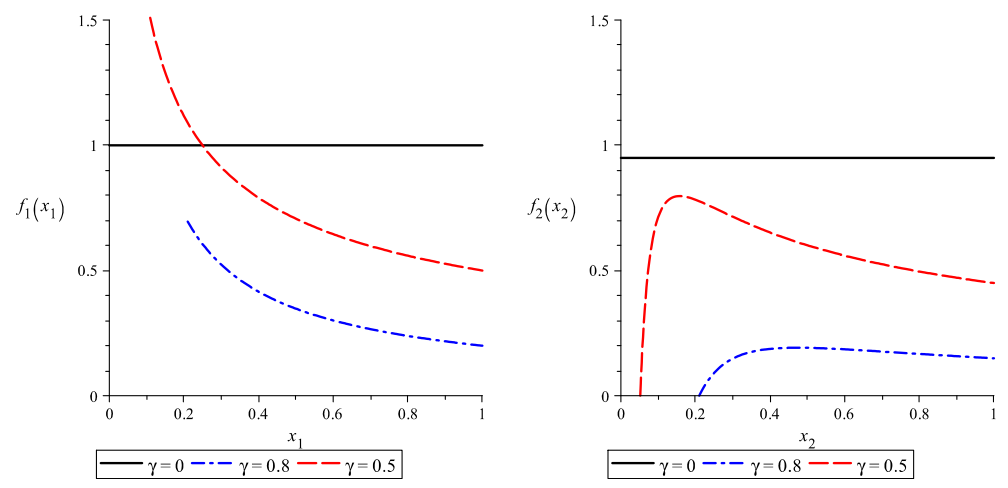

Figure A3: Equilibrium densities for player 1 (left) and 2 (right); $\alpha=1, k=$ 0.95

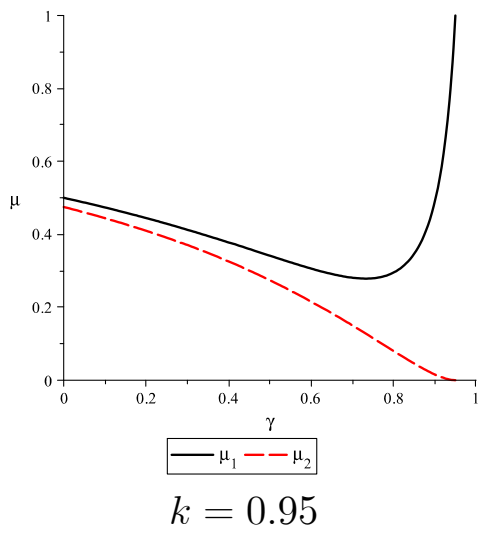

Figure A4: Expected Efforts of players 1 and 2 with $\alpha=1$ 


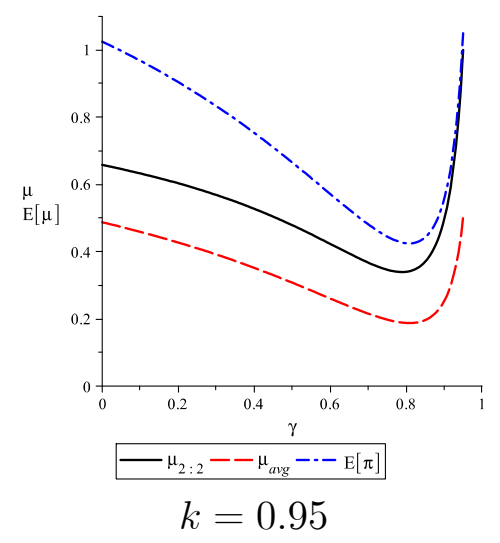

Figure A5: Expected Highest Effort, Expected Average Effort and Expected Average Prize with $\alpha=1$

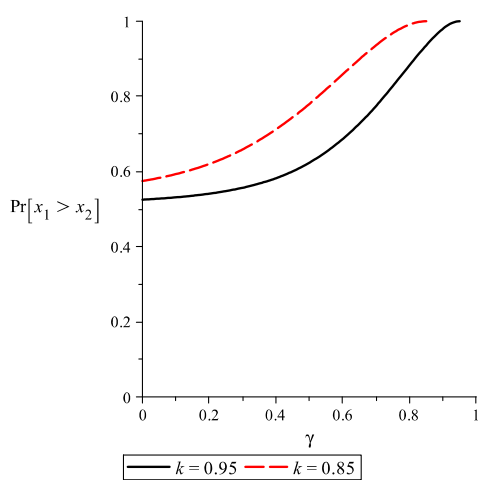

Figure A6: Comparative Statics: Winning Probability of Player 1 for $k=$ 0.95 and $k=0.85$ 


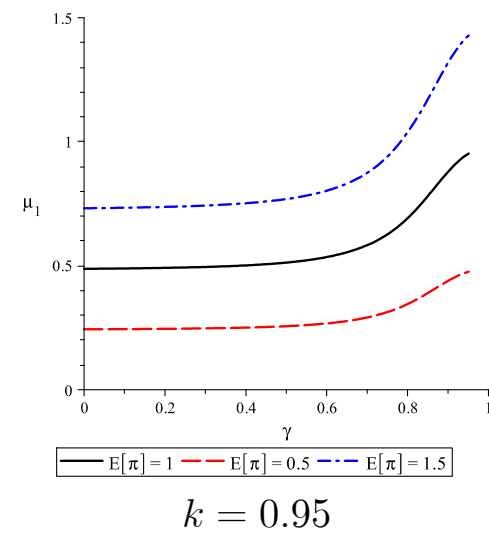

Figure A7: Expected Effort of Player 1 for prize-equivalent APA

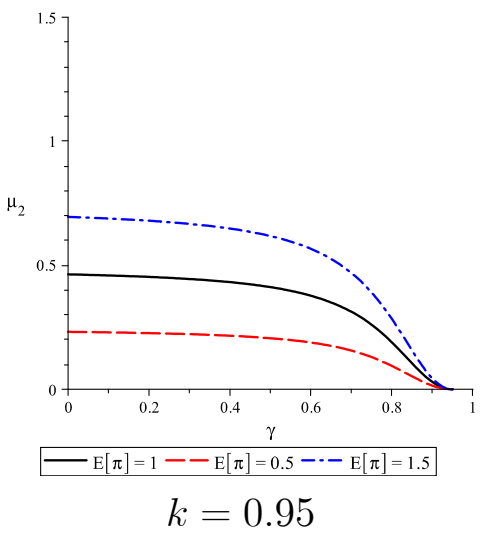

Figure A8: Expected Effort of Player 2 for prize-equivalent APA 